FIAN/TD/16-98

June 1998

\title{
HIGHER-SPIN GAUGE INTERACTIONS FOR MASSIVE MATTER FIELDS IN 3D AdS SPACE-TIME
}

\author{
S. F. Prokushkin I and M. A. Vasiliev f \\ I.E.Tamm Department of Theoretical Physics, Lebedev Physical Institute, \\ Leninsky prospect 53, 117924, Moscow, Russia
}

\begin{abstract}
A remarkable feature of the models with interactions exhibiting higher-spin (HS) gauge symmetries in $d>2$ is that their most symmetric vacua require (anti)-de Sitter (AdS) geometry rather than the flat one. In striking parallelism to what might be expected of $M$ theory HS gauge theories describe infinite towers of fields of all spins and possess naturally space-time SUSY and Chan-Paton type inner symmetries. In this paper, we analyze at the level of the equations of motion the simplest non-trivial HS model which describes HS gauge interactions (on the top of the usual supergravitational and (Chern-Simons) Yang-Mills interactions) of massive spin-0 and spin- $1 / 2$ matter fields in $d=2+1$ AdS space-time. The parameter of mass of the matter fields is identified with the vev of a certain auxiliary field in the model. The matter fields are shown to be arranged into $d 3 N=2$ massive hypermultiplets in certain representations of $U(n) \times U(m)$ Yang-Mills gauge groups. Discrete symmetries of the full system are studied, and the related $N=1$ supersymmetric truncations with $O(n)$ and $S p(n)$ Yang-Mills symmetries are constructed. The simplicity of the model allows us to elucidate some general properties of the HS models. In particular, a new result, which can have interesting implications to the higher-dimensional models, is that our model is shown to admit an "integrating" flow that proves existence of a non-local Bäcklund-Nicolai-type mapping to the free system.
\end{abstract}

\footnotetext{
${ }^{1}$ e-mail: prok@td.lpi.ac.ru

${ }^{2}$ e-mail: vasiliev@td.lpi.ac.ru
} 


\section{Introduction}

The idea that higher-spin (HS) extensions of the ordinary space-time (super)symmetries can drive us to a fundamental unified theory is old enough (see e.g. [1, 2], 3] and references therein). The main problem was [4, 5, 6] how to construct theories with the interactions consistent with HS gauge symmetries. Although some encouraging results have been obtained [7, 8, 9, 10, 11] the problem remained with the gravitational interaction of HS gauge fields [4, 5, 6] as well as with the powerful no-go statements [12, 13] that led to a dominating opinion that consistent HS gauge theories cannot be constructed. A way out was found in [14], where gauge invariant interactions of $d=4$ massless HS gauge fields compatible with gravity have been constructed at the action level in the cubic order in interactions, and in [15], where these results were extended to all orders in interactions at the level of equations of motion (see also [16] for a recent review and further references).

The most important reason which made HS gauge theories invisible within any expansion near the flat space [4, 5, 6] is that HS gauge interactions with unbroken HS gauge symmetries require [14 the cosmological constant to be non-zero because some of the gauge-invariant interactions contain its negative powers. Simultaneously, this property allows one to avoid the no-go statements [12, 13] which claim that HS symmetries cannot be seen in any $S$-matrix constructed in the flat space. It is important to stress that this feature of the HS gauge theories does not mean that the flat background is impossible but rather it means that in the flat background the HS symmetries should necessarily be broken (spontaneously). Together with another important fact that HS gauge theories contain towers of massless fields with infinitely increasing spins (which form multiplets of the HS symmetries) this implies that any spontaneously broken phase of a HS gauge theory should contain infinitely many massive HS excitations similarly to string theories.

Nowadays a unified theory is identified with $M$ theory, a hypothetical theory which reduces to $d=11$ supergravity in the low-energy limit and gives rise to superstring theories by virtue of compactification of extra dimensions. Since superstring theories describe infinite towers of massive HS excitations on the top of a finite number of massless modes it is natural to expect that $M$ theory might be some HS gauge theory in eleven or higher dimensions and that ordinary string theories in ten and lower dimensions result from the originally massless HS gauge fields by means of spontaneous breakdown of the HS gauge symmetries via compactification of extra dimensions. Massless modes of the superstring theory (supergravity) are then expected to correspond to unbroken maximal finite dimensional subalgebras of the original infinite-dimensional HS gauge symmetry algebras. It is important to note that HS gauge theories are fixed almost unambiguously by the gauge symmetry principle.

From the most elaborated $d 4$ example it follows [15, 16] that the ambiguity is basically in the choice of the Yang-Mills (spin -1) gauge group and is parallel to the ambiguity in the Chan-Paton structure. The HS gauge theories contain only two fundamental coupling constants, the gravitational constant $\kappa$ and the cosmological constant $\lambda$. Their dimensionless combination $g^{2} \sim \kappa^{2} \lambda^{2}$ is identified with the Yang-Mills coupling constant (in $d=4$ ). From the $d=3$ analysis of this paper we will learn that some parameters of matter fields (masses) arise as moduli of certain auxiliary scalar fields.

Recently, a remarkable conjecture was made [17, 18, 19, 20, 21] on the large $N$ corre- 
spondence of a fundamental theory in AdS spaces to conformal theories on their boundaries. This conjecture is an important development of the physics of singletons [22, 23]. The degrees of freedom of the singleton boundary models are identified with the maximally supersymmetric conformal Yang-Mills models. A nature of a fundamental bulk theory is more obscure. We believe that this is a HS gauge theory in the AdS space. This idea is somewhat reminiscent of the results of $d 2$ analysis in 24. A similar conjecture on the HS gauge nature of $M$ theory was recently put forward in 25].

The HS symmetry algebras [26] were identified in 27] with the infinite-dimensional Lie superalgebras constructed from the Heisenberg-Weyl algebras with spinorial generating elements and some their further extensions [28, 29] (see sections 3 and 9 for more details). In other words, the HS symmetries are realized as (appropriately supersymmetrized) Moyal star products [30, 31] in certain auxiliary spinor (twistor) spaces. It is interesting that the Moyal product was recently found to be relevant to the $M$ theory in its $\mathrm{M}$ (atrix) formulation [32, 33, 34, 35. A parallelism between HS gauge theories and the Fedosov quantization was recently emphasized in [36]. Another interesting parallelism is due to the analysis of $N=2$ critical open superstring in [37] where it was shown that physical degrees of freedom of the model describe self-dual fields of arbitrary high spin and can naturally be described in terms of a hyperspace with spinor commuting coordinates. Remarkably, these additional coordinates have the same structure as the auxiliary spinor variables used to describe the HS dynamics in terms of the Moyal star product 27.

In this paper, we study the non-linear equations of motion which describe HS interactions of massive matter fields in $2+1$ dimensions. Since HS gauge fields do not propagate in $d 3$ [38, 39, 40], the analysis of the $d 3$ model is simpler than that of the higher-dimensional models with propagating HS fields. The simplicity of the model allows us to make some of the statements on its properties rather explicit. On the other hand, a general character of our conclusions will allow us to speculate how these extend to less trivial models in higher dimensions.

We will demonstrate that the model at hand exhibits a number of natural properties in the context of possible applications to $M$ (string) theory. In particular, we show that the full system possesses naturally $N=2$ (target space) SUSY and describes HS interactions of $d 3$ hypermultiplets. An essential property that fits nicely the superstring picture is that the HS models constructed in this paper admits $U(n)$ Yang-Mills (spin 1) gauge symmetries as well as $N=1$ supersymmetric truncations with orthogonal and symplectic gauge groups (the sector of even spins, which contains gravity and corresponds to closed string, is a part of all HS gauge theories). An important feature of the HS gauge theories studied further in this paper is that consistent HS gauge interactions require non-zero cosmological constant. One of the main conclusions is that the non-local character of the Moyal product together with the presence of a non-zero cosmological constant might imply some sort of non-locality of the HS models at the interaction level because these two properties allow HS interactions with arbitrary high derivatives (but no nonlocalities at the linear level!). This is again in agreement with what one would expect of a model underlying string theory (in this respect the cosmological constant in HS gauge theories plays a role analogous to that of the string tension in superstring theory) and has a striking similarity with the conclusions of the analysis of the noncommutative Matrix models 32, 33, 34, 35]. 
The main result of this paper consists of the explicit construction of non-linear equations of motion which describe interactions of spin-0 and spin- $\frac{1}{2}$ massive matter fields via HS gauge potentials. This model is a generalization of that with massless matter fields proposed in [41]. We show that the parameter of mass of the matter fields appears as the vacuum value of a certain scalar auxiliary field and construct a vacuum solution of the system which is invariant under its discrete symmetries. Another important result consists of the explicit construction of a flow that commutes with the original system and allows one to reduce constructively its solutions to those of the linearized system by virtue of a sort of Bäcklund-Nicolai non-linear map [42]. With the aid of the example of $d 3$ gravity we demonstrate that this mapping should be essentially non-local because the relevant power series in higher derivatives of the original fields are infinite beyond the linearized approximation.

The paper is self-contained and is organized as follows. We start in sect. 2 with the explanation of the formulation of the free field equations in a form of covariant constancy conditions supplemented with appropriate constraints. This formulation follows [43] and is adapted to theories with symmetries mixing higher derivatives of the dynamical fields as it is the case in the HS theories. In sect. 3 we reproduce the main results of 44 on the operator realization of free massive field equations. In sect. 1 an appropriate form of the star (Moyal) product is defined and some of its properties are discussed. In sect. 5 we present the full nonlinear system which describes massive matter fields interacting via HS gauge fields. Some vacuum solutions of this system are presented in sect. 6. In sect. 7 we analyze the linearization of the full system near the vacuum solutions of sect. 6 and show that it reproduces correctly the free field dynamics. In sect. 8 we construct the integrating flow for the full nonlinear system and discuss locality. In sect. 9 we extend the full system to the case with non-Abelian internal symmetries and study reality conditions and truncations. In sect. 10 we study global (super)symmetries of the system. In Appendix A the regularity theorem is proved which guarantees that all manipulations with the star product in this paper are well defined. The details of the general construction of the vacuum solution are considered in Appendix B. In Appendix $\mathrm{C}$ we demonstrate the non-local character of the "integrating" transformations of sect. 8, using the example of Einstein gravity.

\section{Preliminaries}

It is well known [45, 46, 47, 48, 49 that geometry of space-time can be described in terms of the connection 1-forms of an appropriate space-time symmetry algebra $l$. For example vielbein $h_{\nu}{ }^{a}$ and Lorentz connection $\omega_{\nu}^{a b}=-\omega_{\nu}^{b a}$ of the $d$-dimensional spacetime 3 can be identified with the gauge fields of the Poincaré algebra with the generators $P_{a}$ (translations) and $L_{a b}$ (Lorentz rotations),

$$
A=h^{a} P_{a}+\omega^{a b} L_{a b} .
$$

\footnotetext{
${ }^{3} \nu, \mu=0, \ldots,(d-1)$ are indices of 1 -forms while $a, b \ldots=0, \ldots,(d-1)$ are tangent indices that are raised and lowered by the flat Minkowski metrics $\eta^{a b}=\operatorname{diag}(+-\cdots--)$.
} 
The Poincaré curvatures have a form

$$
\begin{gathered}
R_{\mu \nu}{ }^{a b}=\partial_{\mu} \omega_{\nu}{ }^{a b}-\partial_{\nu} \omega_{\mu}{ }^{a b}+\omega_{\mu}{ }^{a}{ }_{c} \omega_{\nu}{ }^{c b}-\omega_{\nu}{ }^{a}{ }_{c} \omega_{\mu}{ }^{c b}, \\
R_{\mu \nu}{ }^{a}=\partial_{\mu} h_{\nu}{ }^{a}-\partial_{\nu} h_{\mu}{ }^{a}+\omega_{\mu}{ }^{a}{ }_{c} h_{\nu}{ }^{c}-\omega_{\nu}{ }^{a}{ }_{c} h_{\mu}{ }^{c} .
\end{gathered}
$$

Assuming that $h_{\nu}{ }^{a}$ is non-degenerate one identifies $R_{\mu \nu}{ }^{a}$ and $R_{\mu \nu}{ }^{a b}$ with the torsion tensor and the Riemann tensor $\mathcal{R}_{\mu \nu}{ }^{a b}$, respectivelyf. The flat geometry is described by the vielbein $h_{\nu}{ }^{a}$ and the Lorentz connection $\omega_{\nu}^{a b}$ obeying the zero-curvature and zero-torsion conditions

$$
R_{\mu \nu}^{a b}=0, \quad R_{\mu \nu}^{a}=0 .
$$

One can choose a solution of (2.4) in the form

$$
h_{\nu}^{a}=\delta_{\nu}^{a}, \quad \omega_{\nu}^{a b}=0 .
$$

Analogously, one can use gauge fields of the AdS algebra $o(d-1,2)$ to describe the geometry of the AdS space-time. Let us consider the $o(d-1,2)$ gauge fields $A_{\mu}^{B C}=-A_{\mu}^{C B}$ (the indices $B, C=0, \ldots, d$ are raised and lowered by the flat metrics $\eta^{B C}=\operatorname{diag}(+-$ $\cdots-+))$ and set $\omega_{\mu}^{a b}=A_{\mu}^{a b}, h_{\mu}{ }^{a}=(\sqrt{2} \lambda)^{-1} A_{\mu}^{a}$ with the conventions $a, b=0, \ldots, d-1$, $B=(b, \cdot)$. Here $\lambda \neq 0$ is some constant. The respective $o(d-1,2)$ gauge curvatures have the form

$$
\begin{gathered}
R_{\mu \nu}{ }^{a b}=\partial_{\mu} \omega_{\nu}{ }^{a b}+\omega_{\mu}{ }^{a}{ }_{c} \omega_{\nu}{ }^{c b}-2 \lambda^{2} h_{\mu}{ }^{a} h_{\nu}{ }^{b}-(\mu \leftrightarrow \nu), \\
R_{\mu \nu}{ }^{a}=\partial_{\mu} h_{\nu}{ }^{a}+\omega_{\mu}{ }^{a}{ }_{c} h_{\nu}{ }^{c}-(\mu \leftrightarrow \nu),
\end{gathered}
$$

which differs from (2.2), (2.3) by the terms proportional to $\lambda^{2}$ on the r.h.s. of (2.6). Again, one can express Lorentz connection $\omega_{\mu}^{a b}$ via vielbein $h_{\mu}{ }^{a}$ with the aid of the constraint $R_{\mu \nu}{ }^{a}=0$ (for the non-degenerate $h_{\mu}^{a}$ ). Substituting $\omega_{\mu}^{a b}=\omega_{\mu}^{a b}(h)$ into (2.6), one can see that the condition $R_{\mu \nu}^{a b}=0$ is equivalent to

$$
\mathcal{R}_{\mu \nu}{ }^{a b}=2 \lambda^{2}\left(h_{\mu}{ }^{a} h_{\nu}{ }^{b}-h_{\nu}{ }^{a} h_{\mu}{ }^{b}\right)
$$

and therefore describes the AdS space-time with radius $(\sqrt{2} \lambda)^{-1} \cdot\left(\mathcal{R} \equiv \mathcal{R}_{\nu \mu}{ }^{\mu \nu}=-2 d(d-\right.$ 1) $\lambda^{2}$.) Note that (2.8) admits solutions both with the regular metrics (hyperboloid) and with the singular ones (black holes) [50]. The analysis below is applicable in the both cases.

Such a formulation of space-time geometry provides a natural starting point for the formulation of dynamics of free matter fields in terms of covariant constancy conditions for appropriate (infinite-dimensional) representations of a chosen space-time symmetry algebra. Namely, this "unfolded formulation" 43] allows one to rewrite free field equations in the form

$$
d C_{i}=A_{i}^{j} \wedge C_{j}
$$

where $d=d x^{\nu} \frac{\partial}{\partial x^{\nu}}$ is the standard space-time exterior differential, with the gauge fields $A_{i}{ }^{j}=A^{a}\left(T_{a}\right)_{i}{ }^{j}$ obeying the zero-curvature conditions

$$
d A^{a}=U_{b c}^{a} A^{b} \wedge A^{c} .
$$

\footnotetext{
${ }^{4}$ We use conventions with $R_{\mu \nu}{ }^{a b}(\omega(h))=\mathcal{R}_{\mu \nu}{ }^{a b}=\mathcal{R}^{a b}{ }_{\mu \nu}=h_{\lambda}{ }^{a} h^{\sigma b} \mathcal{R}^{\lambda}{ }_{\sigma \mu \nu}, \mathcal{R}^{\lambda}{ }_{\sigma \mu \nu}=\partial_{\mu} \Gamma_{\sigma \nu}^{\lambda}+\Gamma_{\sigma \nu}^{\rho} \Gamma_{\rho \mu}^{\lambda}-$ $(\mu \leftrightarrow \nu) . \Gamma_{\sigma \nu}^{\rho}$ is symmetric Christoffel connection defined via $D_{\nu} g_{\mu \rho}=0$, and $\omega(h)$ solves $R_{\mu \nu}{ }^{a}=0$.
} 
Here 1-forms $A^{a}$ are the gauge fields of $l$ or some its extension $g$ (e.g. supersymmetric or HS ones). $C_{i}$ are some $p$-forms which take their values in a certain representation space of $g$. The zero-curvature condition (2.10) is a counterpart of the equations (2.4). The fact that $\left(T_{a}\right)_{i}{ }^{j}$ form some representation $V$ of $l$ guarantees that the compatibility conditions for (2.9) are satisfied. For the simplest cases of matter fields, $C_{i}$ are 0 -forms (cf. the example of a scalar field below). As we will demonstrate, a natural framework for the relevant representations is provided by the Moyal bracket.

Thus, the free field problem consists in finding an appropriate representation of $l$ that leads to the correct field equations. After the equations are rewritten in the "unfolded form", one can write down their general solution in the pure gauge form $A(x)=$ $-g^{-1}(x) d g(x), C(x)=T\left(g^{-1}\right)(x) C_{0}$, where $C_{0}$ is an arbitrary $x$ - independent element of the representation space of $V$. This general solution has a structure of the covariantized Taylor type expansion (see [43] for more details). In the subsequent sections we give a simple realization of the relevant infinite-dimensional representation of the $d 3$ AdS algebra $o(2,2)$.

At the non-linear level, the problem consists in the construction of a non-trivial (nonlinear in $C$ ) deformation of the equations (2.9) and (2.10) which preserves their formal consistency. This is our main concern for the $d 3$ problem considered in this paper. We will follow a method developed previously in [15, 41], which reduces to imposing non-linear constraints in a larger system of the form (2.9), (2.10) and is in many respects analogous to the method of Hamiltonian reduction.

Now let us illustrate how this approach works at the linearized level by the example of the Klein-Gordon equation $\left(\square+M^{2}\right) \phi=0$ for a massive scalar field $\phi$ in the $d$-dimensional flat space-time. We describe the flat space-time geometry by the zero-curvature gauge fields (2.1) of the Poincaré algebra $i s o(d-1,1)$. To describe dynamics of the spin-0 massive field $\phi(x)$ let us introduce an infinite collection of 0 -forms $\phi_{a_{1} \ldots a_{n}}(x)(n=0,1, \ldots)$ that are totally symmetric tensors obeying the constraints

$$
\eta^{b c} \phi_{b c a_{1} \ldots a_{n}}(x)=-M^{2} \phi_{a_{1} \ldots a_{n}}(x),
$$

where $M$ is an arbitrary constant. Then the unfolded formulation of the Klein-Gordon equation is provided by the following infinite chain of equations,

$$
D_{\nu} \phi_{a_{1} \ldots a_{n}}(x)=h_{\nu}^{b} \phi_{b a_{1} \ldots a_{n}}(x) \text {. }
$$

Here $D_{\nu} \phi_{a}=\partial_{\nu} \phi_{a}+\omega_{\nu a}^{b} \phi_{b}$ is the Lorentz-covariant derivative. As we are in the flat space, one can choose the gauge (2.5) and use the ordinary flat derivative $\partial_{\nu}$ instead of $D_{\nu}$. One can easily check that the system (2.12) is formally consistent (i.e. compatible with $D^{2}=0$ ). Differentiation of the constraints (2.11) shows that they are consistent with (2.12) Ð. These properties are equivalent to the fact that the set of zero-forms $\phi_{a_{1} \ldots a_{n}}(x)$ subject to (2.11) spans some representation of the Poincaré algebra.

To show that the system (2.12) with the constraints (2.11) is equivalent to the KleinGordon equation with the mass $M$ let us identify the scalar field $\phi(x)$ with the $n=0$ component of the tower of tensors $\phi_{a_{1} \ldots a_{n}}(x)$. The first two equations in (2.12) read

$$
D_{\mu} \phi=\phi_{\mu}, \quad D_{\mu} \phi_{\nu}=\phi_{\mu \nu} .
$$

\footnotetext{
${ }^{5}$ Moreover, all these constraints for $n>0$ are consequences of (2.11) and (2.12) at $n=0$.
} 
One solves for $\phi_{\nu}$ from these equations and obtains

$$
D_{\mu} D_{\nu} \phi=\phi_{\mu \nu}
$$

Contracting the indices $\mu, \nu$ with the aid of the Minkowski metrics $\eta^{\mu \nu}$ and taking into account (2.11), we get

$$
\left(\square+M^{2}\right) \phi=0 .
$$

All the rest equations in (2.12) express all highest tensors via higher-order derivatives of $\phi(x)$,

$$
\phi_{\mu_{1} \ldots \mu_{n}}=D_{\mu_{1}} \ldots D_{\mu_{n}} \phi
$$

imposing no additional conditions on the dynamical field $\phi(x)$. Thus, we see that the Klein-Gordon equation results from the constraints (2.11). Note that without constraints (2.11) the system (2.12) is equivalent to (2.15) and therefore is dynamically empty. This simple example demonstrates how constraints can impose dynamics.

One can extend this approach to the case of AdS space-time. In this paper, we consider only the case $d=3$ and use the formalism of two-component spinors. We describe the $2+1$ dimensional AdS space in terms of the Lorentz connection one-form $\omega^{\alpha \beta}=d x^{\nu} \omega_{\nu}^{\alpha \beta}(x)$ and dreibein one-form $h^{\alpha \beta}=d x^{\nu} h_{\nu}{ }^{\alpha \beta}(x)$. Here $x^{\nu}$ are space-time coordinates $(\nu=0,1,2)$ and $\alpha, \beta, \ldots=1,2$ are spinor indices which are raised and lowered with the aid of the symplectic form $\epsilon_{\alpha \beta}=-\epsilon_{\beta \alpha}, A^{\alpha}=\epsilon^{\alpha \beta} A_{\beta}, A_{\alpha}=A^{\beta} \epsilon_{\beta \alpha}, \epsilon_{12}=\epsilon^{12}=1$. The one-forms $\omega_{\alpha \beta}$ and $h_{\alpha \beta}$ are symmetric in $\alpha$ and $\beta$. The AdS geometry is described by the equations

$$
\begin{gathered}
d \omega_{\alpha \beta}=\omega_{\alpha \gamma} \wedge \omega_{\beta}^{\gamma}+\lambda^{2} h_{\alpha \gamma} \wedge h_{\beta}^{\gamma}, \\
d h_{\alpha \beta}=\omega_{\alpha \gamma} \wedge h_{\beta}^{\gamma}+\omega_{\beta \gamma} \wedge h_{\alpha}^{\gamma},
\end{gathered}
$$

which have a form of zero-curvature conditions 10 for $l=o(2,2) \sim s p(2) \oplus s p(2)$. For the space-time geometric interpretation of these equations one has to assume that the dreibein $h_{\nu}{ }^{\alpha \beta}$ is a non-degenerate $3 \times 3$ matrix, so that the inverse dreibein $h^{\nu}{ }_{\alpha \beta}$ can be defined [43],

$$
h_{\nu}^{\alpha \beta} h_{\gamma \delta}^{\nu}=\frac{1}{2}\left(\delta_{\gamma}^{\alpha} \delta_{\delta}^{\beta}+\delta_{\delta}^{\alpha} \delta_{\gamma}^{\beta}\right) .
$$

Then, (2.17) reduces to the zero-torsion condition which expresses Lorentz connection $\omega_{\nu}^{\alpha \beta}$ via dreibein $h_{\nu}^{\alpha \beta}$, and (2.16) implies that the Riemann tensor 2 -form $\mathcal{R}_{\alpha \beta}$ satisfies the AdS vacuum condition $\mathcal{R}_{\alpha \beta}=-\lambda^{2} h_{\alpha \gamma} \wedge h_{\beta}{ }^{\gamma}$.

The massive Klein-Gordon and Dirac equations in the AdS space-time take the form

$$
\square C=\left(\frac{3}{2} \lambda^{2}-M^{2}\right) C \quad \text { and } \quad h_{\alpha}^{\nu}{ }^{\beta} D_{\nu} C_{\beta}=\frac{M}{\sqrt{2}} C_{\alpha}
$$

for the spin-0 boson and spin- $\frac{1}{2}$ fermion fields $C(x)$ and $C_{\alpha}(x)$. Here $\square=D^{\mu} D_{\mu}$, where $D_{\mu}$ is the full background covariant derivative containing the symmetric Christoffel connection defined via the metric postulate $D_{\mu} h_{\nu}^{\alpha \beta}=0$. The world indices $\mu, \nu$ are raised and lowered by the metric tensor $g_{\mu \nu}=h_{\mu}{ }^{\alpha \beta} h_{\nu \alpha \beta}$. To reformulate (2.19) in a form of covariant constancy conditions (2.9) we introduce an infinite set of the symmetric multispinors

\footnotetext{
${ }^{6}$ From now on we will use a form of equation that differs from (2.6), (2.7) by $\omega \rightarrow-\omega$.
} 
$C_{\alpha_{1} \ldots \alpha_{n}}$ for all $n \geq 0$. (From now on we will assume total symmetrization of the indices denoted by the same letter and will often use the notation $C_{\alpha(n)}=C_{\alpha_{1} \ldots \alpha_{n}}$ when only a number of indices is important.) As shown in [43], the following two infinite chains of equations,

$$
D C_{\alpha(n)}=h^{\beta \gamma} C_{\beta \gamma \alpha(n)}+n(n-1)\left(\frac{\lambda^{2}}{4}-\frac{M^{2}}{2\left(n^{2}-1\right)}\right) h_{\alpha \alpha} C_{\alpha(n-2)}
$$

for even $n$, and

$$
D C_{\alpha(n)}=h^{\beta \gamma} C_{\beta \gamma \alpha(n)}-\frac{\sqrt{2} M}{n+2} h_{\alpha}{ }^{\beta} C_{\beta \alpha(n-1)}+n(n-1)\left(\frac{\lambda^{2}}{4}-\frac{M^{2}}{2 n^{2}}\right) h_{\alpha \alpha} C_{\alpha(n-2)}
$$

for odd $n$, where $D$ is the background Lorentz-covariant differential,

$$
D C_{\alpha(n)}=d C_{\alpha(n)}+n \omega_{\alpha}^{\gamma} C_{\gamma \alpha(n-1)},
$$

are equivalent respectively to the Klein-Gordon and Dirac equations (2.19) for the lowest components $C$ and $C_{\alpha}$. Similarly to the flat space example considered before, all the rest equations contained in (2.20) and (2.21) either express highest multispinors via highest space-time derivatives of $C$ or $C_{\alpha}$, or reduce to some identities.

\section{Operator Realization for Arbitrary Mass}

Let us now describe an algebraic construction that leads automatically to the correct massive field equations [44] in $d=3$. Following to [29], we introduce "oscillators" $\hat{y}_{\alpha}$ $(\alpha, \beta=1,2)$ obeying the commutation relations

$$
\left[\hat{y}_{\alpha}, \hat{y}_{\beta}\right]=2 i \epsilon_{\alpha \beta}(1+\nu k), \quad k \hat{y}_{\alpha}=-\hat{y}_{\alpha} k, \quad k^{2}=1
$$

where $[a, b]=a b-b a$, and $\nu$ is a free parameter. The operators $\hat{y}_{\alpha}$ and $k$ are treated here as generating elements of the associative algebra $A q(2, \nu)$ (in the notation of [29]), the enveloping algebra of the relations (3.1). We call $k$ Klein operator.

The main property of these oscillators is that the bilinears

$$
T_{\alpha \beta}=\frac{1}{4 i}\left\{\hat{y}_{\alpha}, \hat{y}_{\beta}\right\}
$$

$(\{a, b\}=a b+b a)$ fulfill the standard $s p(2)$ commutation relations

$$
\left[T_{\alpha \beta}, T_{\gamma \delta}\right]=\epsilon_{\alpha \gamma} T_{\beta \delta}+\epsilon_{\beta \delta} T_{\alpha \gamma}+\epsilon_{\alpha \delta} T_{\beta \gamma}+\epsilon_{\beta \gamma} T_{\alpha \delta}
$$

and

$$
\left[T_{\alpha \beta}, \hat{y}_{\gamma}\right]=\epsilon_{\alpha \gamma} \hat{y}_{\beta}+\epsilon_{\beta \gamma} \hat{y}_{\alpha}
$$

for any $\nu$. A specific realization of these oscillators was originally considered by Wigner [51] who addressed a question whether it is possible to modify the oscillator commutation relations in such a way that the relation $\left[H, a^{ \pm}\right]= \pm a^{ \pm}$remains valid. The latter is a particular case of (3.4) with $H=T_{12}$ and $a^{ \pm}=y_{1,2}$. 
The property (3.3) allows us to realize the $o(2,2)$ gravitational connection forms as

$$
W_{g r}(x)=\omega+\lambda \psi h ; \quad \omega=\frac{1}{8 i} \omega^{\alpha \beta}\left\{\hat{y}_{\alpha}, \hat{y}_{\beta}\right\}, \quad h=\frac{1}{8 i} h^{\alpha \beta}\left\{\hat{y}_{\alpha}, \hat{y}_{\beta}\right\}
$$

where $\psi$ is an additional central involutive element,

$$
\psi^{2}=1, \quad\left[\psi, \hat{y}_{\alpha}\right]=0, \quad[\psi, k]=0,
$$

which is introduced to describe the doubling of $s p(2)$ in the $d 3$ AdS algebra $o(2,2) \sim$ $s p(2) \oplus s p(2)$. The generators for the Lorentz rotations (diagonal $s p(2))$ and AdS translations are

$$
L_{\alpha \beta}=\frac{1}{4 i}\left\{\hat{y}_{\alpha}, \hat{y}_{\beta}\right\}, \quad P_{\alpha \beta}=\frac{1}{4 i}\left\{\hat{y}_{\alpha}, \hat{y}_{\beta}\right\} \psi,
$$

while the components in the direct sum $s p(2) \oplus s p(2)$ are spanned by the combinations $L_{\alpha \beta} \pm P_{\alpha \beta}$ projected out by $\frac{1}{2}(1 \pm \psi)$. Now the equations (2.16) and (2.17), which describe the AdS geometry, read

$$
d W_{g r}=W_{g r} \wedge W_{g r} .
$$

Because of the property (3.3) the $\nu$-dependence does not appear explicitly in (3.8).

Let us introduce following to [44] the operator-valued 0-form

$$
C(\hat{y}, k, \psi \mid x)=\sum_{A, B=0,1} \sum_{n=0}^{\infty} \frac{1}{n !} \lambda^{-\left[\frac{n}{2}\right]} C_{\alpha_{1} \ldots \alpha_{n}}^{A B}(x) k^{A} \psi^{B} \hat{y}^{\alpha_{1}} \ldots \hat{y}^{\alpha_{n}}
$$

where $C_{\alpha_{1} \ldots \alpha_{n}}^{A B}$ are totally symmetric multispinors (this implies the totally symmetric (Weyl) ordering with respect to $\hat{y}_{\alpha}$ ). The following two types of equations,

$$
D C^{\text {aux }}=\lambda \psi\left[h, C^{\text {aux }}\right]
$$

and

$$
D C^{d y n}=\lambda \psi\left\{h, C^{d y n}\right\}
$$

where

$$
D C=d C-[\omega, C],
$$

are formally consistent, i.e. the integrability conditions are satisfied as a consequence of the vacuum conditions (3.8). Indeed, (3.10) corresponds to the adjoint action of the space-time algebra $o(2,2)$ (3.7) on the algebra of modified oscillators. The equation (3.11) corresponds to another representation of the space-time symmetry which we call twisted representation. The fact that one can replace commutator by anticommutator in the $h$ dependent term without destroying the formal consistency is a consequence of the property that the AdS algebra possesses an involutive automorphism that changes a sign of the AdS translations. In the particular realization we use it is induced by the automorphism $\psi \rightarrow-\psi$.

There is an important difference between these two representations. The one corresponding to (3.10) decomposes into an infinite direct sum of finite-dimensional representations of $o(2,2)$. Because of the property (3.4) this representation is $\nu$-independent and, therefore, eq. (3.10) at any $\nu$ is equivalent to that with $\nu=0$. The latter was shown in 
43] to describe an infinite set of auxiliary (topological) fields, each carrying no dynamical degrees of freedom (in a topologically trivial situation). On the other hand, the twisted representation is just the infinite-dimensional representation needed for the description of the matter fields [44] (to simplify notations we will sometimes use the symbol $C$ for the twisted representation $\left.C^{d y n}\right)$.

To see this one has to carry out a component analysis of the equations (3.11), which consists of some operator reorderings bringing all terms into the Weyl-ordered form with respect to $\hat{y}_{\alpha}$. It is convenient to replace all operators by their Weyl symbols, so that $C(\hat{y}, k, \psi) \rightarrow C(y, k, \psi)$ according to the rule

$$
C_{\alpha_{1} \ldots \alpha_{n}}^{A B}(x) k^{A} \psi^{B} \hat{y}^{\alpha_{1}} \ldots \hat{y}^{\alpha_{n}} \rightarrow C_{\alpha_{1} \ldots \alpha_{n}}^{A B}(x) k^{A} \psi^{B} y^{\alpha_{1}} \ldots y^{\alpha_{n}}
$$

with

$$
\left[y_{\alpha}, y_{\beta}\right]=0, \quad k y_{\alpha}=-y_{\alpha} k, \quad k^{2}=1, \quad \psi^{2}=1, \quad[\psi, k]=0, \quad\left[\psi, y_{\alpha}\right]=0 .
$$

As a result, (3.11) takes the form

$$
D C(y, k, \psi)=\psi h^{\alpha \beta}\left[a(N) \frac{\partial}{\partial y^{\alpha}} \frac{\partial}{\partial y^{\beta}}+b(N) y_{\alpha} \frac{\partial}{\partial y^{\beta}}+e(N) y_{\alpha} y_{\beta}\right] C(y, k, \psi),
$$

where $D$ is the Lorentz-covariant differential, $D=d-\omega^{\alpha \beta} y_{\alpha} \frac{\partial}{\partial y^{\beta}}$, and $N$ is the Euler operator, $N=y^{\alpha} \frac{\partial}{\partial y^{\alpha}}$. The coefficients $a(n), b(n)$ and $e(n)$ are 44

$$
\begin{aligned}
& a(n)=\frac{i \lambda}{2}\left[1+\nu k \frac{1+(-1)^{n}}{(n+2)^{2}-1}-\frac{\nu^{2}}{(n+2)^{2}\left((n+2)^{2}-1\right)}\left((n+2)^{2}-\frac{1-(-1)^{n}}{2}\right)\right], \\
& b(n)=-\nu k \lambda \frac{1-(-1)^{n}}{2 n(n+2)}, \quad n>0, \quad b(0)=0 \\
& e(n)=-\frac{i \lambda}{2} .
\end{aligned}
$$

Due to the presence of the Klein operator $k$ we have a doubled number of fields compared to the analysis of [43]. The invariant subsets can be projected out with the aid of the projectors $P_{ \pm}$,

$$
C^{ \pm}=P_{ \pm} C, \quad P_{ \pm}=\frac{1 \pm k}{2} .
$$

Then, (3.15) leads to the following two infinite chains of equations for bosons and fermions,

$$
D C_{\alpha(n)}^{ \pm}=\frac{i}{2} \psi\left[\left(1-\frac{\nu(\nu \mp 2)}{(n+1)(n+3)}\right) h^{\beta \gamma} C_{\beta \gamma \alpha(n)}^{ \pm}-\lambda^{2} n(n-1) h_{\alpha \alpha} C_{\alpha(n-2)}^{ \pm}\right]
$$

for even $n$, and

$$
\begin{aligned}
D C_{\alpha(n)}^{ \pm}= & \frac{i}{2} \psi\left(1-\frac{\nu^{2}}{(n+2)^{2}}\right) h^{\beta \gamma} C_{\beta \gamma \alpha(n)}^{ \pm} \pm \psi \frac{\nu \lambda}{n+2} h_{\alpha}{ }^{\beta} C_{\beta \alpha(n-1)}^{ \pm} \\
& -\frac{i}{2} \psi \lambda^{2} n(n-1) h_{\alpha \alpha} C_{\alpha(n-2)}^{ \pm}
\end{aligned}
$$


for odd $n\left(D C_{\alpha(n)}^{ \pm}\right.$is given in (2.22)). These chains of equations differ from (2.20) and (2.21) by some rescalings of the fields $C_{\alpha(n)}$ and lead 44 to the massive field equations (2.19) for the lowest rank components $C$ and $C_{\alpha}$. The values of mass are related to the parameter $\nu$ as follows,

$$
M_{ \pm}^{2}=\lambda^{2} \frac{\nu(\nu \mp 2)}{2}
$$

for bosons and

$$
M_{ \pm}^{2}=\lambda^{2} \frac{\nu^{2}}{2}
$$

for fermions. The signs " \pm " are in agreement with those in (3.19).

The construction above generalizes in a natural way the realization of the equations for massless matter fields in terms of the ordinary $(\nu=0)$ oscillators proposed in 443. An important comment however is that for arbitrary $\nu$ this construction not necessarily leads to non-vanishing coefficients $a(n)$ in (3.15). Consider for instance the equation (3.20) for $C^{+}$(i.e. set $k=1, n=2 m, m \in \mathbf{Z}$ ). We observe that some of the coefficients in front of the first term on the right hand side of (3.20) vanish if $\nu=2 l+1, \forall l \in \mathbf{Z}$. This conclusion is in agreement with the results of [29], where it was shown that for these values of $\nu$ the algebra $A q(2, \nu)$ possesses ideals. Thus, at $\nu=2 l+1$ some of the rescalings of fields mentioned above degenerate and the system of equations derived from the operator realization of (3.11) turns out to be different from that considered in 43. It is important to stress that this does not imply any essential singularity. Rather, for $\nu=2 l+1$ the chains of equations start not from a scalar but from some multispinor component. Interesting enough, the cases of $\nu=1,3$ correspond to the massless electrodynamics [44]. The specificities of the degenerated systems with $\nu=2 l+1$ is a very interesting issue which needs an independent study and will be considered elsewhere. In this paper, we focus on the generic case of the non-degenerate $\nu$.

\section{Star Product}

The generating functions introduced in the next section to describe non-linear $d=3 \mathrm{HS}$ dynamics will be treated as elements of the associative algebra with the following product law,

$$
(f * g)(z, y)=\frac{1}{(2 \pi)^{2}} \int d^{2} u d^{2} v \exp \left(i u_{\alpha} v^{\alpha}\right) f(z+u, y+u) g(z-v, y+v),
$$

where $z_{\alpha}, y_{\alpha}, u_{\alpha}$ and $v_{\alpha}$ are spinor variables. It is easy to see that this product law yields a particular realization of the Heisenberg-Weyl algebra with the defining relations

$$
\left[y_{\alpha}, y_{\beta}\right]_{*}=-\left[z_{\alpha}, z_{\beta}\right]_{*}=2 i \epsilon_{\alpha \beta}, \quad\left[y_{\alpha}, z_{\beta}\right]_{*}=0
$$

$\left([a, b]_{*}=a * b-b * a\right)$. These commutation relations are particular cases of the following simple formulae

$$
\left[y_{\alpha}, f\right]_{*}=2 i \frac{\partial f}{\partial y^{\alpha}}
$$




$$
\left[z_{\alpha}, f\right]_{*}=-2 i \frac{\partial f}{\partial z^{\alpha}}
$$

which are true for an arbitrary $f(z, y)$ (e.g. from the class defined in Appendix A).

With the aid of (4.1) one can check that the operator $\exp \left(i z_{\alpha} y^{\alpha}\right)$ possesses the properties of the inner Klein operator,

$$
e^{i\left(z_{\alpha} y^{\alpha}\right)} * f(z, y)=f(-z,-y) * e^{i\left(z_{\alpha} y^{\alpha}\right)}
$$

for an arbitrary function $f(z, y)$, and

$$
e^{i\left(z_{\alpha} y^{\alpha}\right)} * e^{i\left(z_{\beta} y^{\beta}\right)}=1 .
$$

The star product (4.1) corresponds to the normal ordering of the Heisenberg-Weyl algebra with respect to the generating elements

$$
a_{\alpha}^{+}=\frac{1}{2}\left(y_{\alpha}-z_{\alpha}\right), \quad a_{\alpha}=\frac{1}{2}\left(y_{\alpha}+z_{\alpha}\right),
$$

which satisfy the commutation relations

$$
\left[a_{\alpha}, a_{\beta}\right]_{*}=\left[a_{\alpha}^{+}, a_{\beta}^{+}\right]=0, \quad\left[a_{\alpha}, a_{\beta}^{+}\right]_{*}=i \epsilon_{\alpha \beta}
$$

and are interpreted as creation and annihilation operators. This is most evident from the relations

$$
a_{\alpha}^{+} * f\left(a^{+}, a\right)=a_{\alpha}^{+} f\left(a^{+}, a\right), \quad f\left(a^{+}, a\right) * a_{\alpha}=f\left(a^{+}, a\right) a_{\alpha} .
$$

From (4.8) and (4.9) it follows that

$$
a_{\alpha} f\left(a_{\gamma}^{+} a^{\gamma}\right) * a^{\alpha} f\left(a_{\gamma}^{+} a^{\gamma}\right)=0, \quad a_{\alpha}^{+} f\left(a_{\gamma}^{+} a^{\gamma}\right) * a^{+\alpha} f\left(a_{\gamma}^{+} a^{\gamma}\right)=0
$$

because $a_{\alpha} a^{\alpha}=a_{\alpha}^{+} a^{+\alpha} \equiv 0$.

The formula (4.1) is an integral version [52] of the Moyal star product [30, 31]. The standard differential formulae for the Moyal product can be obtained from (4.1) by the Gaussian integration. The integral formula turns out to be more convenient in many respects.

To formulate the full nonlinear system that possesses all necessary gauge symmetries we will need an associative algebra $\mathcal{A}$ that is generated by the commuting spinors $z_{\alpha}, y_{\alpha}$ $(\alpha=1,2)$,

$$
\left[y_{\alpha}, y_{\beta}\right]=\left[z_{\alpha}, z_{\beta}\right]=\left[z_{\alpha}, y_{\beta}\right]=0 \text {, }
$$

a pair of Clifford elements $\psi_{i}(i=1,2),\left\{\psi_{i}, \psi_{j}\right\}=2 \delta_{i j}$ that commute to all other generating elements, and another pair of Clifford-type elements $k$ and $\rho$ that have the following properties,

$$
k^{2}=1, \rho^{2}=1, k \rho=-\rho k, k y_{\alpha}=-y_{\alpha} k, k z_{\alpha}=-z_{\alpha} k, \rho y_{\alpha}=y_{\alpha} \rho, \rho z_{\alpha}=z_{\alpha} \rho .
$$

Thus, a generic element of $\mathcal{A}$ has a form

$$
\begin{aligned}
& A\left(z, y ; \psi_{1,2}, k, \rho\right)=\sum_{B, C, D, E=0}^{1} \sum_{m, n=0}^{\infty} \frac{1}{m ! n !} A_{\alpha_{1} \ldots \alpha_{m} \beta_{1} \ldots \beta_{n}}^{B C D E} k^{B} \rho^{C} \psi_{1}^{D} \psi_{2}^{E} \\
& \times z^{\alpha_{1}} \ldots z^{\alpha_{m}} y^{\beta_{1}} \ldots y^{\beta_{n}} \text {. }
\end{aligned}
$$


In the sector of spinor variables the product law is defined according to (4.1). Since the elements $\psi_{1}, \psi_{2}, k, \rho$ do not commute, the order of the generating elements is essential. Namely, consider some monomials $f=F(z, y) \Phi\left(\psi_{1,2}, k, \rho\right)$ and $g=G(z, y) \Psi\left(\psi_{1,2}, k, \rho\right)$. Due to the sign changes in (4.12) we have

$$
\Phi\left(\psi_{1,2}, k, \rho\right) G(z, y)=\tilde{G}(z, y) \Phi\left(\psi_{1,2}, k, \rho\right)
$$

with some $\tilde{G}$. The full product is then defined as

$$
(f * g)\left(z, y ; \psi_{1,2}, k, \rho\right)=(F * \tilde{G})(z, y) \Phi\left(\psi_{1,2}, k, \rho\right) \Psi\left(\psi_{1,2}, k, \rho\right) .
$$

Let us remind the reader that invertible mappings $\dagger$ and $\sigma$ of some algebra $\mathcal{F}$ to itself that have the properties

$$
\begin{gathered}
\left(\lambda_{1} a_{1}+\lambda_{2} a_{2}\right)^{\dagger}=\bar{\lambda}_{1} a_{1}^{\dagger}+\bar{\lambda}_{2} a_{2}^{\dagger}, \\
\left(a_{1} * a_{2}\right)^{\dagger}=a_{2}^{\dagger} * a_{1}^{\dagger}, \quad\left(a^{\dagger}\right)^{\dagger}=a,
\end{gathered}
$$

and

$$
\begin{gathered}
\sigma\left(\lambda_{1} a_{1}+\lambda_{2} a_{2}\right)=\lambda_{1} \sigma\left(a_{1}\right)+\lambda_{2} \sigma\left(a_{2}\right), \\
\sigma\left(a_{1} * a_{2}\right)=\sigma\left(a_{2}\right) * \sigma\left(a_{1}\right), \quad \forall a, a_{1}, a_{2} \in \mathcal{F}, \quad \forall \lambda_{1}, \lambda_{2} \in \mathbf{C},
\end{gathered}
$$

are called involution and antiautomorphism, respectively. The algebra $\mathcal{A}$ admits the following important involution $\dagger$ and antiautomorphism $\sigma$ [58, 28,

$$
\begin{gathered}
{\left[A\left(z, y ; \psi_{1,2}, k, \rho\right)\right]^{\dagger}=\bar{A}^{r e v}\left(-z, y ; \psi_{1,2}, k, \rho\right),} \\
\sigma\left[A\left(z, y ; \psi_{1,2}, k, \rho\right)\right]=A^{r e v}\left(-i z, i y ; \psi_{1,2}, k, \rho\right),
\end{gathered}
$$

where the notation $A^{\text {rev }}(\ldots)$ means that an order of all generating elements in the monomial expressions on the r.h.s. of (4.13) is reversed. The properties (4.16)-(4.19) can be easily checked with the aid of (4.1). The important property of the transformations (4.20) and (4.21) is that the inner Klein element $\exp \left(i z_{\alpha} y^{\alpha}\right)$ and the element $K$,

$$
K=k e^{i(z y)}, \quad(z y)=z_{\alpha} y^{\alpha}
$$

are invariant,

$$
\begin{array}{cc}
\left(e^{i(z y)}\right)^{\dagger}=e^{i(z y)}, & K^{\dagger}=K, \\
\sigma\left(e^{i(z y)}\right)=e^{i(z y)}, & \sigma(K)=K .
\end{array}
$$

\section{Nonlinear System with Arbitrary Mass}

To formulate the full nonlinear system that possesses all necessary gauge symmetries and reduces at the linearized level to the free system described in sect. 3 we introduce three types of the generating functions $W, B$, and $S_{\alpha}$ that take values in $\mathcal{A}$ and depend on the space-time coordinates $x^{\nu}(\nu=0,1,2)$. $W$ is a space-time 1-form, $W=d x^{\nu} W_{\nu}\left(z, y ; \psi_{1,2}, k, \rho \mid x\right)$, while $B=B\left(z, y ; \psi_{1,2}, k, \rho \mid x\right)$ and $S_{\alpha}=S_{\alpha}\left(z, y ; \psi_{1,2}, k, \rho \mid x\right)$ are space-time 0 -forms. 
We start with the system of equations analogous to the $d 4$ system of [15] and $d 3$ massless system of 41,

$$
\begin{gathered}
d W=W * \wedge W, \\
d B=W * B-B * W, \\
d S_{\alpha}=W * S_{\alpha}-S_{\alpha} * W, \\
S_{\alpha} * S^{\alpha}=-2 i(1+B * K), \\
S_{\alpha} * B=B * S_{\alpha},
\end{gathered}
$$

where $K$ is given by (4.22).

The multispinorial coefficients $A_{\alpha_{1} \ldots \alpha_{m} \beta_{1} \ldots \beta_{n}}^{B C D E}$ in the expansions (4.13) of the functions $A\left(z, y ; \psi_{1,2}, k, \rho \mid x\right)=W_{\mu}, B$, or $S_{\alpha}$ carry standard Grassmann parity $\pi=0$ or 1 in accordance with the number of spinor indices,

$$
\begin{gathered}
\pi\left(W_{\mu, \alpha(m) \beta(n)}\right)=|m+n|_{2}, \quad \pi\left(B_{\alpha(m) \beta(n)}\right)=|m+n|_{2}, \\
\pi\left(S_{\gamma, \alpha(m) \beta(n)}\right)=|m+n+1|_{2},
\end{gathered}
$$

where

$$
|n|_{2}= \begin{cases}0, & n-\text { even } \\ 1, & n \text { - odd }\end{cases}
$$

and are defined to commute with the generating elements $z_{\alpha}, y_{\alpha}, k, \rho, \psi_{1,2}$. As a result, the commutators on the right hand sides in (5.1)-(5.3) reduce to supercommutators in terms of the polynomials of $z_{\alpha}, y_{\alpha}, k, \rho$ and $\psi_{1,2}$.

With the aid of the obvious involutive automorphism $\rho \rightarrow-\rho$ of $\mathcal{A}$, combined with the symmetry of the equations $S_{\alpha} \rightarrow-S_{\alpha}$, we truncate the system (5.1)-(5.5) to the one with $W$ and $B$ independent of $\rho$ and $S_{\alpha}$ linear in $\rho$,

$$
\begin{gathered}
W\left(z, y ; \psi_{1,2}, k, \rho \mid x\right)=W\left(z, y ; \psi_{1,2}, k \mid x\right), \quad B\left(z, y ; \psi_{1,2}, k, \rho \mid x\right)=B\left(z, y ; \psi_{1,2}, k \mid x\right), \\
S_{\alpha}\left(z, y ; \psi_{1,2}, k, \rho \mid x\right)=\rho s_{\alpha}\left(z, y ; \psi_{1,2}, k \mid x\right) .
\end{gathered}
$$

It is this reduced system which is discussed below and will be shown to describe the dynamics of massive matter fields. Now the gauge fields $W$ and the matter fields $B$ take values in the algebra $A$, the subalgebra of $\mathcal{A}$ spanned by $\rho$-independent elements.

For this system one finds taking into account (4.5), (4.12), and (4.22) that

$$
K * W=W * K, \quad K * B=B * K
$$

and

$$
K * S_{\alpha}=-S_{\alpha} * K .
$$

The additional minus sign in (5.11) is due to the factor of $\rho$ in (5.9). The dynamical system we study in this paper differs from that proposed in 41 by the redefinitions $B \rightarrow-i B * K, S_{\alpha} \rightarrow \rho S_{\alpha}$ and by the doubling of the fields due to the extra dependence on $k\left(k^{2}=1\right)$. As we shall see below, the latter property is essential for the description of massive supersymmetric matter multiplets. 
Let $l$ be the Lie superalgebra constructed from the associative algebra $A$ via (anti)commutators. The equations (5.2) and (5.3) describe 0-forms in the adjoint representation of $l$. Therefore, the system (5.1)-(5.5) contains zero-curvature equations (5.1), covariant constancy conditions (5.2), (5.3) and the constraints (5.4), (5.5) which do not contain space-time derivatives. These constraints make the full system dynamically non-trivial.

The equations (5.1)-(5.5) are general coordinate invariant because of using the exterior algebra formalism and are invariant under the infinitesimal HS gauge transformations

$$
\begin{gathered}
\delta W=d \varepsilon-W * \varepsilon+\varepsilon * W, \\
\delta B=\varepsilon * B-B * \varepsilon, \\
\delta S_{\alpha}=\varepsilon * S_{\alpha}-S_{\alpha} * \varepsilon,
\end{gathered}
$$

where $\varepsilon=\varepsilon\left(z, y ; \psi_{1,2}, k \mid x\right)$ is an arbitrary ( $\rho$-independent) gauge parameter. The main technical problem now consists in elucidating a physical content of these equations by proving that they indeed describe correct relativistic dynamics at the linearized level and beyond. To show this one should first find an appropriate vacuum solution of the equations and then analyze perturbations. These problems are solved in the two subsequent sections.

Before going into technical details, let us show that the form of the constraints (5.4), (5.5) is fixed by the very simple requirement that local Lorentz symmetry remains undeformed in all orders in interactions [53, 54, 55, 56].

The infinitesimal local Lorentz transformations with the parameter $\eta^{\alpha \beta}(x)$ are

$$
\delta f=\left[\eta^{\alpha \beta} L_{\alpha \beta}^{t o t}, f\right]_{*},
$$

where $L_{\alpha \beta}^{t o t}$ are the generators of the inner $s p(2) \sim o(1,2)$ automorphism of the algebra,

$$
L_{\alpha \beta}^{t o t}=\frac{i}{4}\left(\left\{z_{\alpha}, z_{\beta}\right\}_{*}-\left\{y_{\alpha}, y_{\beta}\right\}_{*}\right) .
$$

Actually, with the aid of (4.3) and (4.4) we see that these generators rotate properly the spinor generating elements,

$$
\delta z_{\alpha}=2 \eta_{\alpha}{ }^{\beta} z_{\beta}, \quad \delta y_{\alpha}=2 \eta_{\alpha}{ }^{\beta} y_{\beta} .
$$

Thus, the system (5.1)-(5.5) is explicitly invariant under local Lorentz transformations (5.15). However, this symmetry is spontaneously broken due to the constraint (5.4) because the r.h.s. of (5.4) has a non-vanishing vacuum value and therefore $S_{\alpha}$ itself must have a non-vanishing vacuum value. Therefore, the question is whether there exists another local Lorentz symmetry that rotates properly spinor indices of the dynamical fields and leaves the vacuum solution invariant. Existence of such Lorentz symmetry in all orders in interactions is a non-trivial property which fixes a form of the constraints (5.4), (5.5).

Indeed, because of (5.11) the constraints (5.4), (5.5) have a form of the deformed oscillator algebra (3.1). The elements

$$
M_{\alpha \beta}=\frac{i}{4}\left\{S_{\alpha}, S_{\beta}\right\}_{*}
$$


are analogous to (3.2) and therefore obey the Lorentz commutation relations and rotate properly $S_{\alpha}$,

$$
\left[M_{\alpha \beta}, S_{\gamma}\right]_{*}=\epsilon_{\alpha \gamma} S_{\beta}+\epsilon_{\beta \gamma} S_{\alpha}
$$

Then, we introduce the generators

$$
l_{\alpha \beta}=L_{\alpha \beta}^{t o t}-M_{\alpha \beta}
$$

which also satisfy the $s p(2)$ commutation relations $\square$. Taking into account (5.5), we obtain

$$
\delta B=\left[\eta^{\alpha \beta} l_{\alpha \beta}, B\right]_{*}=\eta^{\alpha \beta}\left[L_{\alpha \beta}^{t o t}, B\right]_{*},
$$

i.e. $l_{\alpha \beta}$ rotate properly the field $B$ which is shown below to describe matter fields.

For the gauge fields $W$ we obtain

$$
\delta W=D\left(\eta^{\alpha \beta} l_{\alpha \beta}\right)=\left(d \eta^{\alpha \beta}\right) l_{\alpha \beta}+\eta^{\alpha \beta}\left[L_{\alpha \beta}^{t o t}, W\right]_{*} .
$$

Here $D(f)=d f-[W, f]_{*}$ and therefore $D\left(\eta^{\alpha \beta}\right)=d\left(\eta^{\alpha \beta}\right)$, since $\eta^{\alpha \beta}(x)$ is independent of any auxiliary variables (i.e. it is proportional to the unit element of $A$ ). Also, $D\left(l_{\alpha \beta}\right)=$ $\left[L_{\alpha \beta}^{t o t}, W\right]_{*}$ because $d L_{\alpha \beta}^{t o t}=0$ and $D M_{\alpha \beta}=0$ (cf. eq.(5.3)). From (5.21) one concludes that the gauge field for a true local Lorentz symmetry is

$$
W_{L}=\omega_{L}^{\alpha \beta} l_{\alpha \beta}
$$

while the other gauge fields are rotated properly under the local Lorentz transformations.

The auxiliary field $S_{\alpha}$ is expressed (up to the gauge ambiguity) via $B$ by the constraint (5.4) (cf. eq.(7.11)). Its transformation law therefore is

$$
\left[\eta^{\alpha \beta} L_{\alpha \beta}^{t o t}, S_{\gamma}(B)\right]_{*}=\eta^{\alpha \beta}\left(\epsilon_{\alpha \gamma} S_{\beta}(B)+\epsilon_{\beta \gamma} S_{\alpha}(B)\right)+\frac{\delta S_{\gamma}}{\delta B} \delta B
$$

where $\delta B=\eta^{\alpha \beta}\left[L_{\alpha \beta}^{t o t}, B\right]_{*}$. Making use of (5.18), we find

$$
\delta S_{\gamma}=\left[\eta^{\alpha \beta} l_{\alpha \beta}, S_{\gamma}\right]_{*}=\frac{\delta S_{\gamma}}{\delta B} \delta B
$$

As a result, the $s p(2)$ local Lorentz rotations induced by $l_{\alpha \beta}$ do not act on the index $\gamma$ of $S_{\gamma}$, acting only on the physical fields $B$.

Thus, the form of the constraints (5.4), (5.5) indeed guarantees that the local Lorentz symmetry remains unbroken.

\section{Vacuum Solutions}

We consider vacuum solutions of the system (5.1)-(5.5) with

$$
B_{0}=\nu
$$

\footnotetext{
${ }^{7}$ This is the standard situation with the diagonal algebra $\left(L_{\alpha \beta}^{t o t}\right)$ of two subalgebras spanned by $l_{\alpha \beta}$ and $M_{\alpha \beta}$.
} 
where $\nu$ is some constant independent of the space-time coordinates and auxiliary variables. As a consequence of (6.1), the vacuum fields $W_{0}, S_{0 \alpha}$ have to satisfy

$$
\begin{gathered}
d W_{0}=W_{0} * \wedge W_{0}, \\
d S_{0}=W_{0} * S_{0 \alpha}-S_{0 \alpha} * W_{0}, \\
{\left[S_{0 \alpha}, S_{0 \beta}\right]_{*}=-2 i \epsilon_{\alpha \beta}(1+\nu K),}
\end{gathered}
$$

while the equations $(5.2)$ and $(5.5)$ are trivially satisfied.

Due to (5.11) we see that (6.4) has a form of the deformed oscillator relations (3.1). For $\nu=0$, the standard choice is $S_{0 \alpha}=\rho z_{\alpha}$. For general $\nu$, a class of solutions of the equations (6.4) is found in Appendix B. Here we describe the following three most important solutions,

$$
S_{0 \alpha}^{ \pm}=\rho\left(z_{\alpha}+\nu\left(z_{\alpha} \pm y_{\alpha}\right) \int_{0}^{1} d t t e^{i t(z y)} k\right)
$$

and

$$
\begin{aligned}
S_{0 \alpha}^{\text {sym }}(z, y)= & \rho z_{\alpha}-\rho \frac{\nu}{8} \int_{-1}^{1} d s(1-s)\left[e^{\frac{i}{2}(s+1)(z y)}\left(y_{\alpha}+z_{\alpha}\right) * \Phi\left(\frac{1}{2}, 2 ;-K \ln |s|^{\nu}\right)\right. \\
& \left.+e^{\frac{i}{2}(s+1)(z y)}\left(y_{\alpha}-z_{\alpha}\right) * \Phi\left(\frac{1}{2}, 2 ; K \ln |s|^{\nu}\right)\right] * K,
\end{aligned}
$$

where $\Phi(a, c ; x)$ is the degenerate hypergeometric function (see Appendix B for more detail) and $K$ and $(z y)$ are defined in (4.22). The ambiguity in the solutions of the equation (6.4) originates from the gauge transformation (5.14). All three solutions $S_{0 \alpha}^{ \pm}$ and $S_{0 \alpha}^{s y m}$ belong to the same gauge equivalence class. It is easy to see that $S_{0 \alpha}^{ \pm}$solve (6.4) by direct insertion into (6.4) (making use of (4.10)). To prove that $S_{0 \alpha}^{\text {sym }}$ solves (6.4) is more tricky (see Appendix B).

The important properties of $S_{0 \alpha}^{ \pm}$and $S_{0 \alpha}^{s y m}$ are

$$
S_{0 \alpha}^{ \pm}(z, y ; k, \rho)=-\bar{S}_{0 \alpha}^{\mp}(-z, y ; k, \rho), \quad S_{0 \alpha}^{ \pm}(z, y ; k, \rho)=i S_{0 \alpha}^{\mp}(-i z, i y ; k, \rho),
$$

and

$$
S_{0 \alpha}^{\text {sym }}(z, y ; k, \rho)=-\bar{S}_{0 \alpha}^{\text {sym }}(-z, y ; k, \rho), \quad S_{0 \alpha}^{\text {sym }}(z, y ; k, \rho)=i S_{0 \alpha}^{\text {sym }}(-i z, i y ; k, \rho) .
$$

The solution $S_{0 \alpha}^{\text {sym }}$ is fixed uniquely by the properties (6.8). In the part of the analysis independent of the particular form of a vacuum solution, the symbol $S_{0 \alpha}$ will be used for any one of the above solutions. However in the analysis of the discrete symmetries and reality conditions in sect. 9 it will be important to use the solution $S_{0 \alpha}^{s y m}$, which is invariant under the corresponding discrete transformations. In fact, the derivation of its explicit form (6.6) is one of the important results of this paper. We expect that it is this solution that is appropriate for the analysis of a locality in the model as discussed in the subsequent sections ?.

\footnotetext{
${ }^{8}$ When some restrictions on the class of transformations are imposed by locality, the statement that different solutions for $S_{0}$ belong to the same gauge equivalence class should be reconsidered.
} 
Now, let us turn to the equation (6.3). Since $d S_{0 \alpha}=0$, we get

$$
\left[W_{0}, S_{0 \alpha}\right]_{*}=0 .
$$

Thus, $W_{0}$ belongs to the subalgebra $A_{S} \subset A$ spanned by elements that commute with $S_{0 \alpha}$, i.e. $A_{S}$ is the centralizer of $S_{0 \alpha}$. For the case of $\nu=0$, from (4.4) it follows that $A_{S}$ is the subalgebra of functions independent of $z$. To find $A_{S}$ for general $\nu$ we construct the elementary generating elements $\tilde{y}_{\alpha}$ commuting with $S_{0 \alpha}^{ \pm}$(6.5) or $S_{0 \alpha}^{\text {sym }}$ (6.6). The final result is

$$
\begin{gathered}
\tilde{y}_{\alpha}^{ \pm}(z, y)=y_{\alpha}+\nu\left(z_{\alpha} \pm y_{\alpha}\right) \int_{0}^{1} d t(t-1) e^{i t(z y)} k \\
\tilde{y}_{\alpha}^{s y m}(z, y)=y_{\alpha}+k \frac{\nu}{8} \int_{-1}^{1} d s(1-s) \exp \left\{\frac{i}{2}(s+1)(z y)\right\}\left[\left(y_{\alpha}+z_{\alpha}\right) \Phi\left(\frac{1}{2}, 2 ;-k \ln |s|^{\nu}\right)\right. \\
\left.-\left(y_{\alpha}-z_{\alpha}\right) \Phi\left(\frac{1}{2}, 2 ; k \ln |s|^{\nu}\right)\right] .
\end{gathered}
$$

Remarkably, $\tilde{y}_{\alpha}$ again obey the commutation relations of the form (3.1),

$$
\left[\tilde{y}_{\alpha}, \tilde{y}_{\beta}\right]_{*}=2 i \epsilon_{\alpha \beta}(1+\nu k), \quad \tilde{y}_{\alpha} k=-k \tilde{y}_{\alpha} .
$$

It is easy to check that $\left[\tilde{y}_{\alpha}^{ \pm}, S_{0 \beta}^{ \pm}\right]_{*}=0$. The fact that $\left[\tilde{y}_{\alpha}^{s y m}, S_{0 \beta}^{s y m}\right]_{*}=0$ is less trivial (see Appendix B). Note that as a by-product we have found that the deformed oscillator algebra can be realized in terms of the embedding into the tensor product of two Heisenberg-Weyl algebras equipped with the generating element $k$.

Since $k, \psi_{1}$, and $\psi_{2}$ commute with $S_{0 \alpha}$, the subalgebra $A_{S}$ is spanned by the power series of $\tilde{y}_{\alpha}, \psi_{1}, \psi_{2}$, and $k$, i.e. its generic element has the form

$$
A_{S}\left(z, y ; \psi_{1,2}, k\right)=\sum_{B, C, D=0}^{1} \sum_{n=0}^{\infty} \frac{1}{n !} A_{S \alpha_{1} \ldots \alpha_{n}}^{B C D} k^{B} \psi_{1}^{C} \psi_{2}^{D} \tilde{y}^{\alpha_{1}} * \ldots * \tilde{y}^{\alpha_{n}},
$$

where $A_{S} \alpha_{1} \ldots \alpha_{n}$ are totally symmetric multispinors (i.e. we choose the Weyl ordering). Note that due to the presence of $\psi_{1}$ and $\psi_{2}, A_{S}$ is isomorphic to $A q(2, \nu) \otimes \operatorname{Mat}_{2}(\mathbf{C})$. According to (6.9), $W_{0}$ should have a form (6.13).

Since the commutation relations (6.12) have a form of the deformed oscillator algebra (3.1), one can use the properties (3.2)-(3.4) to build a solution of the vacuum equations (6.2). Namely, we can choose $W_{0}$ in the form analogous to (3.5),

$$
W_{0}=\omega_{0}+\lambda h_{0} \psi_{1}, \quad \omega_{0}=\frac{1}{8 i} \omega_{0}^{\alpha \beta}\left\{\tilde{y}_{\alpha}, \tilde{y}_{\beta}\right\}_{*}, \quad h_{0}=\frac{1}{8 i} h_{0}^{\alpha \beta}\left\{\tilde{y}_{\alpha}, \tilde{y}_{\beta}\right\}_{*} .
$$

Then (6.2) leads to the zero-curvature and zero-torsion conditions (2.16), (2.17) for $\omega_{0}^{\alpha \beta}$ and $h_{0}^{\alpha \beta}$, thus describing AdS background geometry, so that the fields $\omega_{0}^{\alpha \beta}$ and $h_{0}^{\alpha \beta}$ can be interpreted, respectively, as the background AdS Lorentz connection and dreibein of sect. 2. This completes construction of the background solution.

Let us emphasize that the form of the constraint (5.4) leads in a rather non-trivial way to the AdS background geometry via realization of the vacuum centralizer $A_{S}$ in terms of the deformed oscillators $\tilde{y}_{\alpha}$. 


\section{Linearization}

Now we study the system (5.1)-(5.5) perturbatively expanding it near the vacuum solution (6.1), (6.6), and (6.14) as

$$
B=B_{0}+B_{1}+\ldots, \quad S_{\alpha}=S_{0 \alpha}+S_{1 \alpha}+\ldots, \quad W=W_{0}+W_{1}+\ldots .
$$

This gives in the first order

$$
\begin{gathered}
D_{0} W_{1}=0, \\
D_{0} C=0, \\
D_{0} S_{1 \alpha}=\left[W_{1}, S_{0 \alpha}\right]_{*}, \\
{\left[S_{0 \alpha}, S_{1}^{\alpha}\right]_{*}=-2 i C * K,} \\
{\left[S_{0 \alpha}, C\right]_{*}=0,}
\end{gathered}
$$

where we denote $C=B_{1}$, and $D_{0}$ is the background covariant adjoint differential, $D_{0} P=$ $d P-W_{0} \wedge P+(-)^{r} P \wedge W_{0}$ for a $r$-form $P$.

From (7.6), we see that $C$ takes its values in $A_{S}$, i.e. $C=C\left(\tilde{y} ; k, \psi_{1,2}\right)$. Inserting

$$
C=C^{a u x}\left(\psi_{1}\right)+C^{d y n}\left(\psi_{1}\right) \psi_{2}
$$

into (7.3), one arrives at

$$
\begin{aligned}
& D_{0}^{L} C^{\text {aux }}=\psi_{1} \lambda\left[h_{0}, C^{\text {aux }}\right]_{*}, \\
& D_{0}^{L} C^{d y n}=\psi_{1} \lambda\left\{h_{0}, C^{d y n}\right\}_{*},
\end{aligned}
$$

where $D_{0}^{L}$ is the Lorentz covariant differential,

$$
D_{0}^{L} C=d C-\left[\omega_{0}, C\right]_{*} .
$$

The equations (7.8) and (7.9) coincide with (3.10) and (3.11), respectively. Because the commutation relations for the generating elements $\tilde{y}_{\alpha}$ have the form $(6.12)$, from the results of sect. 3 it follows that the equations $(7.3)$ and (17.6) lead in the sector of $C^{d y n}$ to the Klein-Gordon and Dirac equations for spin-0 and spin- $\frac{1}{2}$ fields with the masses (3.22), (3.23). As before, the rank-0 and 1 components of the expansion (6.13) for $C^{d y n}$ are identified with physical scalar and spinor matter fields respectively.

As mentioned in sect. 3, the equations (7.8) for the fields $C^{\text {aux }}$ describe an infinite set of auxiliary (topological) fields, each carrying no dynamical degrees of freedom (locally).

As a next step, we determine $S_{1 \alpha}$ from (7.5). It is not difficult to integrate (7.5) perturbatively. We however will apply a useful trick exploiting the possibility to vary the free parameter $\nu$. (All generating functions acquire a non-trivial dependence on $\nu$ via the dependence on the deformed oscillators $\tilde{y}(6.10)$.) The advantage of this approach is that it works for any vacuum solution $S_{0 \alpha}$, while the resulting concrete formulae look very differently for different solutions like $S_{0 \alpha}^{ \pm}\left(\underline{6.5}\right.$ ) and $S_{0 \alpha}^{s y m}(\underline{6.6})$ and sometimes are not too illuminating.

With the aid of (6.4) and (7.6) one can easily check that

$$
S_{1 \alpha}=C * \frac{\partial S_{0 \alpha}}{\partial \nu}+\left[S_{0 \alpha}, \chi\right]_{*}
$$


is a solution of (7.5) with an arbitrary function $\chi\left(z, y ; \psi_{1,2}, k\right)$. This ambiguity is a manifestation of the gauge freedom (5.14) at the linearized level with $\varepsilon_{1}=-\chi$. We therefore gauge fix $\chi=0$ by setting

$$
S_{1 \alpha}=C * \frac{\partial S_{0 \alpha}}{\partial \nu}
$$

By substituting this solution into (7.4) and making use of (6.9), (7.3), and (7.6), we get

$$
\left[C * \frac{\partial}{\partial \nu} W_{0}, S_{0 \alpha}\right]_{*}=\left[W_{1}, S_{0 \alpha}\right]_{*},
$$

and, therefore,

$$
W_{1}=\omega(\tilde{y} ; \psi, k)+C * \frac{\partial W_{0}}{\partial \nu},
$$

where an arbitrary one-form $\omega\left(\tilde{y} ; \psi_{1,2}, k\right)$ takes values in $A_{S}$. It is this one-form $\omega\left(\tilde{y} ; \psi_{1,2}, k\right)$ that is identified with the generating function for the HS gauge fields. The substitution of (7.14) into (7.2) gives

$$
D_{0} \omega=-D_{0}\left(C * \frac{\partial W_{0}}{\partial \nu}\right)
$$

The evaluation of the r.h.s. of this formula is analogous to the derivation of (7.13) and leads to

$$
D_{0}\left(C * \frac{\partial W_{0}}{\partial \nu}\right)=C * \frac{\partial}{\partial \nu}\left(d W_{0}-W_{0} * \wedge W_{0}\right)=0
$$

according to (6.2). Thus we conclude that

$$
D_{0} \omega=d \omega-W_{0} * \wedge \omega-\omega * \wedge W_{0}=0
$$

i.e. there is no contribution to the r.h.s. of the HS strengths in the first order in $C$.

The first order corrections to the HS strengths have been studied for the case of massless matter fields with $\nu=0$ in 41], where it was shown that such corrections are trivial (can be compensated by some field redefinitions). We generalize this conclusion to the case of an arbitrary $\nu$. This is of course a desired result because the right hand sides of the Chern-Simons dynamical equations for HS gauge fields can acquire corrections due to some currents constructed from the matter fields, but there is no reason to expect nontrivial currents linear in physical fields. We therefore conclude that, as expected, in the first order the system (5.1)-(5.5) describes free matter fields in some background of the vacuum gauge fields (AdS background for the simplest vacuum (6.14)). This completes the analysis of the linearized equations. Two comments are now in order.

The first is that at any order the perturbative analysis of the system will reduce to some $\frac{\partial}{\partial x^{\mu}}$ and $\left[S_{0}, \ldots\right]_{*}$ equations of the type (7.2)-(7.6) with the r.h.s.-s determined via solutions in the lowest orders. This system is overdetermined but consistent due to the general consistency properties of the full system with respect to farther $\frac{\partial}{\partial x^{\mu}}$ differentiations and taking the commutators $\left[S_{\alpha}, \ldots\right]_{*}$. Some specificities (but no inconsistencies) can only take place for the degenerate points $\nu=2 l+1, l \in \mathbf{Z}$ discussed in sect. 3 and Appendix B.

The second comment is that the r.h.s.-s are always well defined. The point is that our formulae contain some non-polynomial expressions in the variables $y_{\alpha}$ and $z_{\beta}$ (cf. (6.5), 
(6.10) ), and it is not guaranteed a priori that $*$-products of such expressions do not contain divergencies. The fact that all perturbative computations are well defined is based on the theorem proved in [53], which guarantees the regularity of all our expressions provided that the original variables belong to a certain class of regular functions. In Appendix A, we reformulate this theorem in the form appropriate to the analysis in this paper and show that $S_{0 \alpha}, \tilde{y}_{\alpha}$ and any functions of $\tilde{y}_{\alpha}$ of the type (6.13) belong to the appropriate regularity class.

\section{Non-Local Integrating Mapping}

A remarkable property of the non-linear system (5.1)-(5.5) is that it admits a flow that allows one to express constructively its solutions in terms of those of the linearized problem. This construction is a generalization of the field redefinitions (7.12), (7.14) used in sect. 7 to show that HS gauge field strengths do not have nontrivial sources linear in fields.

We introduce a parameter $\tau$ as an additional evolution parameter. The meaning of $\tau$ is that it serves as an expansion parameter in powers of interactions, i.e. as an effective coupling constant. The idea is that the evolution flow in $\tau$ will allow us to express the dynamical fields of the non-linear problem with $\tau \neq 0$ via those of the free problem with $\tau=0$. Let us note that this method has some similarity with the method of evolution over a coupling constant developed in [57] in a different context.

We therefore assume that $W=W(\tau), B=B(\tau)$ and $S_{\alpha}=S_{\alpha}(\tau)$ and introduce a shifted variable $\mathcal{B}(\tau)$,

$$
B(\tau)=\nu+\tau \mathcal{B}(\tau)
$$

where $\nu$ is some constant parameter, the vacuum value of the field $B$, while $\mathcal{B}$ is assumed to be a fluctuational part. The system (5.1)-(5.5) acquires the form

$$
\begin{gathered}
d W=W * \wedge W, \\
d \mathcal{B}=W * \mathcal{B}-\mathcal{B} * W, \\
d S_{\alpha}=W * S_{\alpha}-S_{\alpha} * W, \\
S_{\alpha} * S^{\alpha}=-2 i(1+\nu K+\tau \mathcal{B} * K), \\
S_{\alpha} * \mathcal{B}=\mathcal{B} * S_{\alpha} .
\end{gathered}
$$

Note that the parameter $\tau$ falls out of all the equations except for (8.5).

Now one observes that for the limiting case $\tau=0$, the system (8.2)-(8.6) reduces to the free one. Indeed, setting

$$
\nu=B_{0}, \quad \mathcal{B}(\tau=0)=B_{1} \equiv C, \quad W(\tau=0)=W_{0} \equiv \omega, \quad S_{\alpha}(\tau=0)=S_{0 \alpha},
$$

we see that at $\tau=0$ the system (8.2)-(8.6) has the form of the vacuum system (6.1)- (6.4) plus the linear equations (7.3), (7.6) for the matter fields $C$. Hence, after $S_{0 \alpha}$ is excluded, we arrive at the free system

$$
\begin{gathered}
d \omega=\omega * \wedge \omega \\
d C=\omega * C-C * \omega,
\end{gathered}
$$


where $\omega=\omega(\tilde{y})$ and $C=C(\tilde{y})$ belong to $A_{S}$. Let us note that the situation here is similar to that with contractions of Lie algebras. For all values of $\tau \neq 0$, the systems of equations (8.2)-(8.6) are pairwise equivalent since the field redefinition (8.1) is non-degenerate. On the other hand, although the field redefinition (8.1) degenerates at $\tau=0$, the system of equations (8.2)-(8.6) still makes sense, describing the free field dynamics.

Let us now define a flow with respect to $\tau$,

$$
\begin{gathered}
\frac{\partial W}{\partial \tau}=(1-\mu) \mathcal{B} * \frac{\partial W}{\partial \nu}+\mu \frac{\partial W}{\partial \nu} * \mathcal{B}, \\
\frac{\partial \mathcal{B}}{\partial \tau}=(1-\mu) \mathcal{B} * \frac{\partial \mathcal{B}}{\partial \nu}+\mu \frac{\partial \mathcal{B}}{\partial \nu} * \mathcal{B}, \\
\frac{\partial S_{\alpha}}{\partial \tau}=(1-\mu) \mathcal{B} * \frac{\partial S_{\alpha}}{\partial \nu}+\mu \frac{\partial S_{\alpha}}{\partial \nu} * \mathcal{B},
\end{gathered}
$$

where $\mu$ is an arbitrary parameter. By applying $\frac{\partial}{\partial \tau}$ to the both sides of eqs.(8.2)-(8.6) one concludes that for any $\mu$ the system (8.9)-(8.11) is compatible with (8.2)-(8.6). Therefore, solving the system (8.9)-(8.11) with the initial data

$$
\mathcal{B}(\tau=0)=C, \quad W(\tau=0)=\omega, \quad S_{\alpha}(\tau=0)=S_{0 \alpha},
$$

we can express solutions of the full nonlinear system at $\tau=1$ via solutions of the free system at $\tau=0$. This approach is very efficient at least perturbatively and allows one to derive order by order the relevant field redefinitions. In particular, it is easy to see that at $\mu=0$ it leads directly to the solution (7.12) used in sect. 7 to show that the HS gauge field strengths do not admit non-trivial sources linear in fields. Note that even at the linearized level it is a complicated technical problem to find a form of an appropriate solution without using the flow (8.9)-(8.11).

The flows (8.9)-(8.11) at different $\mu$ develop within the same gauge equivalence class. To see that any variation of $\mu$ is induced by some gauge transformation one should find a gauge parameter $\varepsilon$ such that

$$
\frac{\partial W}{\partial \mu}=D \varepsilon, \quad \frac{\partial \mathcal{B}}{\partial \mu}=[\varepsilon, \mathcal{B}]_{*}, \quad \frac{\partial S_{\alpha}}{\partial \mu}=\left[\varepsilon, S_{\alpha}\right]_{*}, \quad \varepsilon(\tau=0)=0,
$$

where $D \varepsilon=d \varepsilon-[W, \varepsilon]_{*}$. The compatibility condition of (8.13) with (8.9)-(8.11) is satisfied if

$$
\frac{\partial \varepsilon}{\partial \tau}=\frac{\partial \mathcal{B}}{\partial \nu}+(1-\mu) \mathcal{B} * \frac{\partial \varepsilon}{\partial \nu}+\mu \frac{\partial \varepsilon}{\partial \nu} * \mathcal{B},
$$

which condition just fixes a $\tau$-dependence of $\varepsilon$. Thus, one is free to choose any value of $\mu$. There are three most interesting cases: $\mu=0$ (left), $\mu=1$ (right) and $\mu=\frac{1}{2}$ (symmetric).

Important comment is that one has to be careful in making statements on the locality of the mapping induced by the flow (8.9)-(8.11). Indeed, although it does not contain explicitly space-time derivatives, it contains them implicitly via highest components $C_{\alpha(n)}$ of the generating function $C(\tilde{y})$, which are identified with the highest derivatives of the matter fields by the equations (8.8) (see the chains of equations (3.20), (3.21) which are 
the component forms of (8.8)). For example, the equation (8.10) at $\mu=0$ in the zero order in $\tau$ reads

$$
\frac{\partial}{\partial \tau} \mathcal{B}_{1}(z, y)=C(\tilde{y}) * \frac{\partial C(\tilde{y})}{\partial \nu}
$$

Because of using the $*$-product, for each fixed rank multispinorial component of the l.h.s. of this formula there appears, in general, an infinite series involving bilinear combinations of the components $C_{\alpha(n)}$ with all $n$ on the r.h.s. of (8.15). Therefore, the r.h.s. of (8.15) effectively involves all order space-time derivatives, i.e. the transformation laws (8.9)- 8.11) can effectively describe some non-local transformations. This means that we cannot consider the system (5.1)-(5.5) as locally equivalent to the free system (8.7), (8.8). Instead we can only claim that there exists a non-local mapping between the free and nonlinear system. This mapping is reminiscent of the Nicolai mapping in supersymmetric models 42 and the Bäcklund mapping in the integrable systems.

At the linearized level, however, the transformations induced by the integrating flow (8.9)-(8.11) turn out to be local for the following simple reason. In this case, all field redefinitions are linear in the matter fields $C$. They can contain HS gauge fields $\omega$, but in the linear approximation only the vacuum (zero-order) part of the gauge fields can contribute. This part is nothing else as the background gravitational 1-forms (6.14) which are bilinear in the auxiliary variables $\tilde{y}_{\alpha}$. As a result, the transformations for physical fields, induced by (8.9), are always polynomial in $\tilde{y}_{\alpha}$. So, they can contain at most a finite number of the coefficients $C_{\alpha(n)}$. This is equivalent to the statement that the linearized field transformations contain only a finite number of space-time derivatives and therefore are local. Thus, the statement that the equations of motion for HS fields do not acquire sources linear in the matter fields is the well defined local statement. This is not expected to be the case for the second-order analysis which should cover the case of bilinear HS currents constructed from the matter fields. To illustrate this point we consider in Appendix $\mathrm{C}$ an example of $d 3$ gravity interacting with some matter source.

\section{Extended Symmetries and Truncations}

\subsection{Inner Symmetries}

The HS dynamical systems admit a natural extension to the case with non-Abelian internal (Yang-Mills) symmetries, as was first discovered in [58, 28] for the $d 4$ case 9. The key observation is that the system (5.1)-(5.5) remains consistent if components of all fields take their values in an arbitrary associative algebra $M$ with a unit element $I_{M}$, i.e. the fields $W, B$, and $S_{\alpha}$ take their values in the algebra $\mathcal{A}^{\text {ext }}=\mathcal{A} \otimes M$, where $\mathcal{A}$ is the associative algebra with the general element (4.13). The extended HS algebra $A^{\text {ext }}$ is then identified with the $\rho$-independent part of $\mathcal{A}^{\text {ext }}$, while the fields $S_{\alpha}$ are assumed as before to be linear in $\rho$. The algebra $\mathcal{A}$ admits a natural embedding $\mathcal{A} \rightarrow \mathcal{A} \otimes I_{M}$. The gravitational sector is associated with $A \sim A \otimes I_{M}$ and therefore commutes with $M \sim I_{A} \otimes M$, where $I_{A}$ is the unit element of $A$. Therefore, $M$ describes internal symmetries in the model. So far, we have considered the simplest Abelian case. Now we turn to a general situation with

\footnotetext{
${ }^{9}$ Recently, a particular case of this extension [27] was used in [25] to describe $d 4 N=8$ HS supergravity.
} 
finite-dimensional inner symmetries. For the case of semisimple finite-dimensional inner symmetries, the only possibility is to identify $M$ with some matrix algebra. It is convenient to start with the complex algebra $M=\operatorname{Mat}_{n}(\mathbf{C})$, imposing the reality conditions afterwards.

Thus we set

$$
\begin{gathered}
W\left(z, y ; \psi_{1,2}, k\right) \rightarrow W_{i}{ }^{j}\left(z, y ; \psi_{1,2}, k\right), \quad B\left(z, y ; \psi_{1,2}, k\right) \rightarrow B_{i}{ }^{j}\left(z, y ; \psi_{1,2}, k\right), \\
S_{\alpha}\left(z, y ; \psi_{1,2}, k, \rho\right) \rightarrow S_{\alpha, i}{ }^{j}\left(z, y ; \psi_{1,2}, k, \rho\right),
\end{gathered}
$$

where the matrix indices $i, j=1 \ldots n$ do not affect Grassmann properties (5.6), (5.7).

The vacuum solutions (6.1), (6.5), (6.6), 6.14) give rise to the vacuum solutions of the extended system via embedding $\mathcal{A} \rightarrow \mathcal{A} \otimes I_{M}$, i.e.

$$
W_{0, i}{ }^{j}=W_{0} \delta_{i}{ }^{j}, \quad B_{0, i}{ }^{j}=B_{0} \delta_{i}{ }^{j}, \quad S_{0 \alpha, i}{ }^{j}=S_{0 \alpha} \delta_{i}{ }^{j}
$$

The infinite-dimensional symmetry algebra $l^{\text {ext }}$ of the model is the Lie superalgebra defined via supercommutators in $A^{e x t}=A \otimes \operatorname{Mat}_{n}(\mathbf{C})$. More precisely, we need a real form of the original complex Lie superalgebra $l^{\text {ext }}$ that is compatible with the unitarity of the model. Let us denote this real superalgebra as $h u(n \mid 4)$, where $n$ indicates the factor of $\operatorname{Mat}_{n}(\mathbf{C})$ in the original algebra $A^{\text {ext }}$, while 4 is for the four generating elements $z_{\alpha}, y_{\alpha}$.

The appropriate reality conditions can be imposed with the aid of the involution defined as the mapping (4.20), when acting on $\mathcal{A}$, and as an ordinary Hermitian conjugation on $\operatorname{Mat}_{n}(\mathbf{C})$,

$$
\left(a_{i}{ }^{j}\right)^{\dagger}=\bar{a}_{j}{ }^{i}
$$

This uniquely defines some involution $\dagger$ of the full algebra $\mathcal{A}^{\text {ext }}$. Then, the real HS Lie superalgebra $h u(n \mid 4)$ is extracted by imposing the condition" $\square$

$$
a^{\dagger}=-i^{\pi(a)} a
$$

on $a \in l^{e x t}$, where $\pi=0$ or 1 is the boson-fermion parity given by (5.6).

The appropriate (anti)hermiticity conditions for the variables $W, B, S_{\alpha}$ consistent with the system (5.1)-(5.5) read

$$
W^{\dagger}=-W, \quad S_{\alpha}^{\dagger}=-S_{\alpha}, \quad B^{\dagger}=B,
$$

where an additional sign factor for $B$ is due to the factor of $i$ on the r.h.s. of (5.4). In these formulae, it is assumed that $\dagger$ is also the involution of the Grassmann algebra in which the components of the fields $W, B, S_{\alpha}$ take their values, i.e. it reverses an order of Grassmann factors.

Note that this definition of the reality conditions is compatible with the vacuum solution $B_{0}, W_{0}\left(\tilde{y}_{\alpha}^{s y m}\right.$ ) and $S_{0 \alpha}^{s y m}$ (cf. (6.6), 6.11)), but not with the solutions with $S_{0 \alpha}^{+}$and $S_{0 \alpha}^{-}$which are conjugated to each other. Therefore, from now on we choose $S_{0 \alpha}=S_{0 \alpha}^{s y m}$.

\footnotetext{
${ }^{10}$ More details on the relationship between involutions, conjugations and real forms of complex (super)algebras can be found in [27].
} 


\subsection{Truncations by Automorphisms}

Now we study consistent truncations of the extended system (5.1)-(5.5) induced by its various involutive symmetries. Let $\eta$ be some involutive symmetry of the system (5.1)(5.5), i.e. $\eta^{2}=I$. Then the truncation conditions are $\square$

$$
\eta\left(A_{i}\right)=A_{i}, \quad A_{i}=\left(W_{\mu}, B, S_{\alpha}\right) .
$$

Such a truncation is consistent in the standard sense that every solution of the truncated system is a solution of the full system. Indeed, it is equivalent to setting to zero the variables $A_{i}^{\text {odd }}$ from the odd subspace, $\eta\left(A_{i}^{\text {odd }}\right)=-A_{i}^{\text {odd }}$.

In this section, we consider the truncations of the system (5.1)-(5.5) induced by automorphisms of the associative algebra $\mathcal{A}^{\text {ext }}$. The following automorphisms of $\mathcal{A}^{\text {ext }}$ will be important,

$$
\begin{gathered}
f\left[a\left(z, y ; \rho, k, \psi_{1,2}\right)\right]=a\left(-z,-y ;-\rho, k, \psi_{1,2}\right), \\
\phi\left[a\left(z, y ; k, \psi_{1}, \psi_{2}\right)\right]=a\left(z, y ; k, \psi_{1},-\psi_{2}\right),
\end{gathered}
$$

and the similarity transformation $\kappa$ acting on $(n+m) \times(n+m)$ matrices $M$ as follows,

$$
\kappa(M)=U M U^{-1}, \quad U=\left(\begin{array}{cc}
I_{n} & 0 \\
0 & -I_{m}
\end{array}\right) .
$$

Following to [28 we will use the algebra $h u(n+m \mid 4)$ as a starting point for the derivation of a series of algebras realized in various HS models that can be obtained by virtue of truncations of the equations (5.1)-(5.5). The mappings (9.6), (9.7) and (9.8) are mutually commuting involutive automorphisms of $A^{\text {ext }}$ and, as a consequence, of $h u(n+m \mid 4)$. One can consider their compositions $\phi^{\alpha} f^{\beta} \kappa$ with $\alpha, \beta=0$ or 1 , which are involutive automorphisms as well [2]. These automorphisms induce involutive symmetries of the full system (5.1)-(5.5). The corresponding truncation conditions are

$$
\phi^{\alpha} f^{\beta} \kappa(W)=W, \quad \phi^{\alpha} f^{\beta} \kappa\left(S_{\gamma}\right)=S_{\gamma}, \quad \phi^{\alpha} f^{\beta} \kappa(B)=B
$$

Let us stress that we confine ourselves to only the truncations that do not affect the vacuum solutions (6.1), (6.6), (6.14) since otherwise the resulting truncated equations will not admit a perturbative interpretation (this is why we do not consider the automorphism similar to (9.7) that changes a sign of $\psi_{1}$ ).

Now let us analyze the gauge symmetry algebras of the truncated systems that are spanned by the elements $a$ satisfying the conditions

$$
\phi^{\alpha} f^{\beta} \kappa(a)=a, \quad a \in h u(n+m \mid 4) .
$$

First of all one observes that the truncated algebra acquires an involutive central element $\psi_{1}^{\alpha} k^{\beta} U$ which commutes to every element satisfying (9.10). This means that the truncated algebra decomposes into a direct sum of two subalgebras projected out by the projectors

$$
\mathcal{P}^{ \pm}=\frac{\left(1 \pm \psi_{1}^{\alpha} k^{\beta} U\right)}{2}, \quad \mathcal{P}^{ \pm} l^{ \pm}=l^{ \pm}
$$

\footnotetext{
${ }^{11}$ The involutivity condition is not essential. This is just a particular case of gauging some discrete symmetry group $\Gamma$. In our case $\Gamma$ will be a product of some $Z_{2}$ factors.

${ }^{12}$ The truncations based on the automorphisms of the form $\phi^{\alpha} f^{\beta}$ are not interesting since they eliminate either all fermions or dynamical matter bosons, or all the dynamical matter degrees of freedom.
} 
Let the corresponding subalgebras $l^{ \pm}$be called $h u_{\alpha \beta}^{ \pm}(n, m \mid 4)$. The full system of equations also decomposes into two independent (mutually non-interacting) subsystems singled out by the conditions $\mathcal{P}^{ \pm}\left(A_{i}^{ \pm}\right)=A_{i}^{ \pm}, A_{i}^{ \pm}=\left(W_{\mu}^{ \pm}, B^{ \pm}, S_{\alpha}^{ \pm}\right)$. We will refer to these subsystems as based on $h u_{\alpha \beta}^{ \pm}(n, m \mid 4)$.

The case $\alpha=\beta=0$ is trivial with $h u_{00}^{+}(n, m \mid 4) \sim h u(n \mid 4)$ and $h u_{00}^{-}(n, m \mid 4) \sim$ $h u(m \mid 4)$.

The case $\alpha=0, \beta=1$ is similar to the $d 4$ algebras considered in [28]. The algebras $h u_{01}^{+}(n, m \mid 4) \sim h u_{01}^{-}(m, n \mid 4)$ are spanned by the matrices from $h u(n+m \mid 4)$ with bosons in the diagonal blocks $n \times n$ and $m \times m$, and fermions in the off-diagonal blocks $n \times m$ and $m \times$ $n$. The Klein operator $k$ should be replaced by the matrix $U$, according to the projection conditions (9.11). As a result we arrive at the two-parametric set of consistent models with HS gauge symmetry algebras that are the subalgebras of $h u(n+m \mid 4)$ extracted by the condition (9.10) and the projectors $\frac{(1 \pm k U)}{2}$. In notations of [28] these algebras are $h u(2 n, 2 m \mid 4)$ ㅍ.

The case $\alpha=1, \beta=0$ is analogous to the massless case considered in 41] (where the Klein operator did not appear). The corresponding algebras $h u_{10}^{+}(n, m \mid 4) \sim h u_{10}^{-}(m, n \mid 4)$ are spanned by the matrices from $h u(n+m \mid 4)$ with $\psi_{2}$-independent diagonal blocks $n \times n$ and $m \times m$, and linear in $\psi_{2}$ off-diagonal blocks $n \times m$ and $m \times n$. For these algebras, $\psi_{1}$ should be replaced by the matrix $U$ according to the form of the projector (9.11). The field $\mathrm{C}(7.7)$ acquires the form $\left(\begin{array}{ll}C^{\text {aux }} & C^{\text {dyn }} \\ C^{\text {dyn }} & C^{\text {aux }}\end{array}\right)$, while the background fields become $W_{0}=\omega_{0} I+\lambda h_{0} U$, where $I$ is the $(n+m) \times(n+m)$ unit matrix.

The mixed case $\alpha=1, \beta=1$ can be described analogously. The elements of the algebras $h u_{11}^{+}(n, m \mid 4) \sim h u_{11}^{-}(m, n \mid 4)$ are the matrices from $h u(n+m \mid 4)$ with $\psi_{2}$-independent bosons and linear in $\psi_{2}$ fermions in the diagonal blocks $n \times n$ and $m \times m$, and with $\psi_{2^{-}}$ independent fermions and linear in $\psi_{2}$ bosons in the off-diagonal blocks $n \times m$ and $m \times n$. In this case, $\psi_{1}$ should be replaced by $k U$, in accordance with the projection condition (9.11).

In all three non-trivial cases with $\alpha+\beta \neq 0$, the isomorphisms $h u_{\alpha \beta}^{ \pm}(n, m \mid 4) \sim$ $h u_{\alpha \beta}^{ \pm}(m, n \mid 4) \sim h u_{\alpha \beta}^{\mp}(m, n \mid 4)$ take place. For this reason, in what follows the signs " $\pm "$ will be omitted. In general, algebras with different pairs $n, m$ and $\alpha, \beta(\alpha+\beta \neq 0)$ are pairwise non-isomorphic and lead to the different HS models. At $n=m$, all these algebras are isomorphic to the original algebra, $h u_{\alpha \beta}(n, n \mid 4) \sim h u(n \mid 4), \alpha, \beta=0$ or 1 [4.

The Yang-Mills subalgebras of the infinite-dimensional algebras described above are spanned by the elements independent of $z_{\alpha}, y_{\alpha}$ and $\psi_{2}$ (to ensure commutativity with the space-time symmetry algebra $o(2,2)(6.14)$. For all three non-trivial cases with $\alpha+\beta \neq 0$, the resulting Yang-Mills subalgebra is $u(n) \oplus u(n) \oplus u(m) \oplus u(m)$ (the doubling is due to the presence of either $\psi_{1}$ or $\left.k,\left(\left(\psi_{1}\right)^{2}=k^{2}=1\right)\right)$.

\footnotetext{
${ }^{13}$ The doubling of $n$ and $m$ is due to the presence of the elements $\psi_{1,2}$ which generate the Clifford algebra isomorphic to the $2 \times 2$ matrix algebra.

${ }^{14}$ One can make sure of this taking into account that the doublings of elements of the algebras due to the operators $k$ and $\psi_{1}$ turn out to be equivalent to the doubling of the matrix blocks for even and odd elements separately, with appropriate replacements $k \rightarrow U$ or $\psi_{1} \rightarrow U$, or $k \psi_{1} \rightarrow U$.
} 


\subsection{Truncation by Antiautomorphism}

As shown in [58, 28 for the $d 4$ case and in [41] for the $d 3$ case, further truncations of the extended systems that lead to the orthogonal and symplectic Yang-Mills algebras can be constructed with the aid of antiautomorphisms of the original associative algebra $\mathcal{A}^{\text {ext }}$. Every antiautomorphism $\sigma$ of $A^{\text {ext }} \subset \mathcal{A}^{\text {ext }}$ (i.e. a mapping with the properties (4.18), (4.19) ) induces some automorphism $\tau$ of the Lie superalgebra $l^{\text {ext }}$ (and of $h u_{\alpha \beta}(n, m \mid 4)$ ), according to the following general rule

$$
\tau(a)=-i^{\pi(a)} \sigma(a)
$$

(for more details on the relationship between antiautomorphisms and automorphisms of Lie superalgebras see e.g. [27]). A subalgebra of $h u_{\alpha \beta}(n, m \mid 4)$ can now be extracted by the condition $\tau(a)=a$.

To define some antiautomorphism $\sigma$ on $\mathcal{A}^{\text {ext }}=\mathcal{A} \otimes \operatorname{Mat}_{n}(\mathbf{C})$ it suffices to define it separately on $\mathcal{A}$ and $\operatorname{Mat}_{n}(\mathbf{C})$. We define $\sigma$ on $\mathcal{A}$ according to (4.21). To define how $\sigma$ acts on $\operatorname{Mat}_{n+m}(\mathbf{C})$ one can use the fact that antiautomorphisms $\sigma^{h}$ of $M_{a t m}(\mathbf{C})$ are induced by non-degenerate bilinear forms $h(x, y)=H^{i j} x_{i} y_{j}$ on some representation space of $\operatorname{Mat}_{n+m}(\mathbf{C}), h(x, M y)=h\left(\sigma^{h}(M) x, y\right)$, i.e.

$$
\sigma^{h}\left(M_{i}^{j}\right)=H^{j k} M_{k}^{l} H_{l i}^{-1}, \quad \forall M \in \operatorname{Mat}_{n+m}(\mathbf{C}) .
$$

Let us assume that

$$
H=\left(\begin{array}{cc}
H_{n} & 0 \\
0 & H_{m}
\end{array}\right), \quad H_{n}^{i j}= \pm H_{n}^{j i}, \quad H_{m}^{i j}= \pm H_{m}^{j i} .
$$

The block-diagonal form of $H$ will be important to guarantee that the vacuum solution satisfies the truncation condition. It is important to note that $n \times n$ and $m \times m$ blocks $H_{n}$ and $H_{m}$ are required to have the same symmetry properties 10

The automorphism $\tau$ gives rise to the symmetry $\eta$ of the full system,

$$
\begin{gathered}
\eta\left(W_{\mu}\right)=-i^{\pi(W)} \sigma\left(W_{\mu}\right), \\
\eta(B)=i^{\pi(B)} \sigma(B), \\
\eta\left(S_{\alpha}\right)=i^{\pi(S)+1} \sigma\left(S_{\alpha}\right),
\end{gathered}
$$

where $\pi=0$ or 1 is given by (5.6), (5.7). It is assumed here that $\sigma$ does not affect the Grassmann field coefficients (i.e. the order of the Grassmann factors is not reversed). The truncation is then performed via imposing the condition (9.5). It is important that these conditions leave invariant the vacuum solutions $B_{0} \otimes I_{M}\left(\right.$ (6.1), $S_{0 \alpha}^{s y m} \otimes I_{M}$ (6.6) and $W_{0}\left(\tilde{y}_{\alpha}^{s y m}\right) \otimes I_{M}(6.14)$.

In the spin-1 sector $W_{\mu}^{Y M}$ (i.e. a part of $W_{\mu}$ independent of $z, y$, and $\psi_{2}$ ), the condition $\eta\left(W_{\mu}\right)=W_{\mu}$ reads $\sigma^{h}\left(W_{\mu}^{Y M}\right)=-W_{\mu}^{Y M}$ and means that the fields $W_{\mu}^{Y M}$ correspond to

\footnotetext{
${ }^{15}$ In agreement with the analysis of the $d 4$ case in [28], relaxing this condition does not lead to new possibilities. Indeed, at $\alpha+\beta \neq 0$, the choice of $H_{n}$ and $H_{m}$ with different types of symmetry leads to $\tau^{2}=\kappa(9.8)$. This is equivalent to setting $\kappa=1$ in (9.9) and gives rise to the degenerate systems truncated by the automorphisms $\phi^{\alpha} f^{\beta}$ discussed in the footnote in subsect. 9.2.
} 
the subalgebra that leaves the (skew)symmetric bilinear form $h$ invariant. Thus, the truncations induced by the antiautomorphisms $\sigma$ reduce the unitary Yang-Mills subalgebras to either orthogonal algebras $o(n) \oplus o(n) \oplus o(m) \oplus o(m)$ (H is symmetric) or symplectic algebras $u s p(n) \oplus u s p(n) \oplus u s p(m) \oplus u s p(m)$ ( $H$ is skewsymmetric and $n$ and $m$ are even).

Let us denote the respective subalgebras of $h u_{\alpha \beta}(n, m \mid 4)$ as $h o_{\alpha \beta}(n, m \mid 4)$ and $h u s p_{\alpha \beta}(n, m \mid 4)$, in analogy with the notation of [28]. It is worth to mention that the role of the antiautomorphism $\sigma$ is analogous to that of the twist (orientation) operator in the superstring theory [59].

\section{Global (Super)Symmetries}

\subsection{Global Symmetries}

The system (5.1)-(5.5) is explicitly invariant under the HS gauge transformations (5.12)(5.14). Fixation of the vacuum solution (6.1), (6.6), (6.14) breaks this local symmetry down to some global symmetry, the symmetry of the vacuum. This global symmetry is also a symmetry of the linearized system (7.2)-(7.6) and, as a consequence, of the equations (7.8), (7.9). It is generated by the parameter $\varepsilon_{g l}(x)$ obeying the conditions

$$
\begin{gathered}
d \varepsilon_{g l}=\left[W_{0}, \varepsilon_{g l}\right]_{*}, \\
{\left[\varepsilon_{g l}, S_{0 \alpha}\right]_{*}=0,}
\end{gathered}
$$

which follow from the requirements that $\delta W_{0}=0$ and $\delta S_{0 \alpha}=0$ ( $\delta B_{0}=0$ holds trivially), i.e. $\varepsilon_{g l}$ belongs to the stability subalgebra of the vacuum solution $\square$.

The condition (10.2) implies that $\varepsilon_{g l}$ belongs to $A_{S}^{e x t}=A_{S} \otimes M a t_{n}(\mathbf{C})$, i.e. $\varepsilon_{g l}$ is of the form (6.13) with the matrix-valued coefficients. The equation (10.1) fixes a dependence of $\varepsilon_{g l}$ on the space-time coordinates $x^{\mu}$ in terms of the initial data $\varepsilon_{g l}\left(x_{0}\right)=\varepsilon_{g l}^{0}$ at any space-time point $x_{0}$ (in some neighborhood of $x_{0}$ ).

According to (6.13), $A_{S}^{\text {ext }}$ contains elements linear in $\psi_{2}$. From the definition (7.7), it follows that the symmetry parameters linear in $\psi_{2}$ mix the auxiliary fields $C^{\text {aux }}$ and the dynamical fields $C^{d y n}$. As mentioned in sect. 3, $C^{\text {aux }}$ describes an infinite set of the auxiliary fields, each having at most a finite number of degrees of freedom. This means that $C^{\text {aux }}$ decomposes into a sum of finite-dimensional representations of the spacetime symmetry algebra $o(2,2)$. Since $o(2,2)$ is non-compact, it has no non-trivial finitedimensional unitary representation and, therefore, these modes cannot survive in any unitary theory. In terms of solutions of the field equations the point is that the auxiliary fields do not admit non-trivial solutions after imposing appropriate boundary conditions at space infinity. Thus we conclude that the unitarity requirement rules out the symmetry with the parameters $\varepsilon_{g l}$ linear in $\psi_{2}$. So, similarly to the $d=4$ case [60, we will analyze the subalgebra $l^{g}$ of the full global symmetry superalgebra that is spanned by the $\psi_{2^{-}}$ independent elements $\square$ and admits unitary representations.

\footnotetext{
${ }^{16}$ Here it is important to note that, due to the zero-curvature conditions (6.2), general coordinate transformations of the vacuum solution reduce to some gauge transformations according to the relations $\delta_{\xi} A_{\nu}=D_{\nu} \varepsilon+\xi^{\mu} R_{\mu \nu}$, where $\delta_{\xi}$ is an infinitesimal diffeomorphism with a parameter $\xi^{\mu}$ and $\varepsilon=\xi^{\mu} A_{\mu}$.

${ }^{17}$ One can factor out a trivial center spanned by constant parameters $\varepsilon_{g l}$.
} 
Since $l^{g}$ does not contain $\psi_{2}$, the element $\psi_{1}$ becomes central. As a result, $l^{g}$ decomposes into a direct sum of the two subalgebras with the aid of the projectors $\frac{1}{2}\left(1 \pm \psi_{1}\right)$, i.e. $l^{g}=l^{g+} \oplus l^{g-}$. As $\psi_{1}$ is a coefficient in front of the dreibein in (6.14), for the linearized equations to admit sensible flat limit one has to require that the two components $l^{g+}$ and $l^{g-}$ be isomorphic to each other. Let us now analyze what global symmetry algebras result from the gauge algebras $h u_{\alpha \beta}(n, m \mid 4), h o_{\alpha \beta}(n, m \mid 4)$ and $h u s p_{\alpha \beta}(n, m \mid 4)$ defined in the previous section.

In the case $\beta=0$ the two choices of $\alpha$ lead to different results. The case with $\alpha=1$ is more general. From the projection condition (9.11) one easily finds that the resulting global symmetry algebra $h u_{0}(n, m \mid 2)=h u_{0}(n, 0 \mid 2) \oplus h u_{0}(0, m \mid 2)$, i.e. it has a form $\left(\begin{array}{cc}A & 0 \\ 0 & B\end{array}\right)$ with $n \times n$ and $m \times m$ diagonal blocks $A$ and $B$ which depend on the spinor variables $\tilde{y}$ and $k$. According to (9.11), $\psi_{1}$ is identified here with the matrix $U=\left(\begin{array}{cc}1 & 0 \\ 0 & -1\end{array}\right)$. Therefore the dreibein is also proportional to $U$ and the flat limit is possible only in the case with $n=m$ when left and right sectors are symmetric. It is easy to see that if one starts with the case $\alpha=0$ then the resulting global algebra is either $h u_{0}(n, n \mid 2)$ or $h u_{0}(m, m \mid 2)$ depending on what sign is chosen in (9.11). Note that this class of algebras is analogous to that considered in 441] for the $d=3$ massless case.

In the case of $\beta=1$ one arrives at the same result for the both choices of $\alpha$. Here $k$ is identified with $\pm U$ according to (9.11), while $\psi_{1}$ should be kept as an independent generating element. Therefore the resulting algebra $h u_{1}(n, m \mid 2)$ is left-right symmetric and the corresponding linearized equations of motion admit sensible flat limit for all $n$ and $m$. Here the bosonic symmetries live in the diagonal blocks while the fermionic ones in the off-diagonal blocks. These algebras are analogous to the $d=4$ global symmetry algebras considered in [28].

The algebras $h o_{\beta}(n, m \mid 2)$ and $h u s p_{\beta}(n, m \mid 2)$ are defined analogously.

\section{$10.2 \quad N=2$ SUSY}

In the case of the Abelian internal symmetry, the maximal finite-dimensional subalgebra of $l^{g}$ contains the superalgebra $\operatorname{osp}(2,2) \oplus \operatorname{osp}(2,2)$ spanned by generators $\Pi_{ \pm} T^{A}$, where

$$
\Pi_{ \pm}=\frac{1 \pm \psi_{1}}{2}
$$

and $T^{A}=\left\{T_{\alpha \beta}, Q_{\alpha}^{(1)}, Q_{\alpha}^{(2)}, J\right\}$ with

$$
T_{\alpha \beta}=\frac{1}{4 i}\left\{\tilde{y}_{\alpha}, \tilde{y}_{\beta}\right\}_{*}, \quad Q_{\alpha}^{(1)}=\tilde{y}_{\alpha}, \quad Q_{\alpha}^{(2)}=\tilde{y}_{\alpha} k, \quad J=k+\nu .
$$

The fact 61 that these generators form $\operatorname{osp}(2,2)$ is a simple consequence of the properties of the deformed oscillator algebra (3.1). Thus, the system (5.1)-(5.5) possesses $N=2$ global SUSY for arbitrary $\nu$.

Now let us consider the extended systems. We start with the case $\beta=1$. One can see that only the case $n=m$ corresponds to the supersymmetric theory. At $n \neq$ $m$, the algebras $h u_{1}(n, m \mid 2)$ do not contain the AdS SUSY subalgebras. Indeed, the 
generators of $\operatorname{osp}(2,2)$ (10.4) can be embedded into these algebras as $T_{\alpha \beta} \otimes I_{M}, J \otimes I_{M}$ and $Q_{\alpha}^{(1)} \otimes Y_{M}, Q_{\alpha}^{(2)} \otimes Y_{M}$, where

$$
Y_{M}=\left(\begin{array}{cc}
0 & A \\
B & 0
\end{array}\right), \quad Y_{M}^{2}=I_{M}
$$

This is possible only at $n=m$.

The case $\beta=0$ turns out to be supersymmetric at any $n$ and $m$ because the generators of $\operatorname{osp}(2,2)$ are embedded into $h u_{0}(n, m \mid 2)$ as $T^{A} \otimes I_{M}$.

To find the maximal $N=2$ supersymmetric finite-dimensional global symmetry subalgebras one should single out those Yang-Mills symmetries of sect. 9 that commute with $o s p(2,2) \oplus \operatorname{osp}(2,2)$. For the case $\beta=1$ and $n=m$, this is $u(n) \oplus u(n)$, the diagonal subalgebra spanned by the $\psi_{1}$ - dependent matrices (the doubling is due to $\psi_{1}$ ). The maximal finite-dimensional subalgebra is therefore $\operatorname{osp}(2,2) \oplus \operatorname{osp}(2,2) \oplus u(n) \oplus u(n)$.

In the case $\beta=0$ with arbitrary $n, m$, the block-diagonal constant matrices commute with $\operatorname{osp}(2,2) \oplus \operatorname{osp}(2,2)$ and, therefore, again the maximal finite-dimensional subalgebras are $\operatorname{osp}(2,2) \oplus \operatorname{osp}(2,2) \oplus u(n) \oplus u(m)$.

Now let us analyze how maximal finite-dimensional global symmetries act on the matter fields. We start with the Abelian case. As we learned from sect. 7 , the equation of motion for the dynamical components $C^{d y n}\left(\tilde{y} ; k, \psi_{1} \mid x\right) \psi_{2}$ in (7.7) leads to the four infinite chains (3.20) for the bosonic components and four infinite chains (3.21) for the fermionic components. Each chain realizes some infinite-dimensional representations of the AdS algebra $o(2,2)=o(2,1) \oplus o(2,1)$ and describes a single particle. With respect to $N=2$ SUSY $\operatorname{osp}(2,2) \oplus \operatorname{osp}(2,2)$, all bosonic and fermionic components of $C^{d y n}$ belong to the same supermultiplet. This follows from the following expansion of $C^{d y n}\left(\tilde{y} ; k, \psi_{1}\right)$,

$$
C^{d y n}\left(\tilde{y} ; k, \psi_{1}\right)=\left[C_{+}^{0}(\tilde{y})+C_{-}^{0}(\tilde{y})\right]+\left[C_{+}^{1}(\tilde{y})+C_{-}^{1}(\tilde{y})\right] \psi_{1},
$$

where in accordance with (3.19) $C_{ \pm}^{0,1}=P_{ \pm} C^{0,1}, P_{ \pm}=\frac{1 \pm k}{2}$, and from the realization (10.4) of the generators of $\operatorname{osp}(2,2) \oplus \operatorname{osp}(2,2)$. Thus we arrive at the $N=2$ hypermultiplet constituted by 4 scalar and 4 spinor fields,

$$
\left\{C_{+}^{0}(x), C_{-}^{0}(x), C_{+}^{1}(x), C_{-}^{1}(x), C_{+\alpha}^{0}(x), C_{-\alpha}^{0}(x), C_{+\alpha}^{1}(x), C_{-\alpha}^{1}(x)\right\} .
$$

The masses are given by the formulae (3.22) and (3.23) for scalars and spinors respectively (the signs \pm in (3.22) and (3.23) correspond to those in (10.6)). The doubling of fields of the same mass is due to the presence of $\psi_{1}$ and is typical for $N=2$ hypermultiplets. In the complexified system the irreducible subsystems are singled out by the conditions $\Pi^{ \pm} C^{ \pm}=C^{ \pm}$with the projector (10.3). However, because the matter fields carry the factor of $\psi_{2}$ according to (7.7), $C^{+}$and $C^{-}$turn out to be conjugated to each other according to (9.4). Another way to see this complex structure is to observe that the similarity transformation $C \rightarrow e^{i \alpha \psi_{1}} C e^{-i \alpha \psi_{1}}$ is equivalent to $C^{ \pm} \rightarrow e^{ \pm 2 i \alpha} C^{ \pm}$.

A generalization to the non-Abelian case is straightforward. For the case $h u_{0}(n, m \mid 2)$ the matter fields arrange into $2(n \otimes \bar{m} \oplus m \otimes \bar{n})$ representation 18 of the global inner

\footnotetext{
${ }^{18}$ In this paper we use the notations of type $n \otimes \bar{m} \oplus m \otimes \bar{n}$ to denote a complex field $\phi_{i}{ }^{j^{\prime}}(i=$ $\left.1, \ldots, n, \quad j^{\prime}=1, \ldots, m\right)$ and its complex conjugate $\bar{\phi}_{j^{\prime}}{ }^{i}$.
} 
symmetry algebra $u(n) \oplus u(m)$. Similarly, in the supersymmetric case $h u_{1}(n, n \mid 2)$ the matter fields belong to the $2\left(n \otimes \bar{n}^{\prime} \oplus \bar{n} \otimes n^{\prime}\right)$ representation of the global inner symmetry algebra $u(n) \oplus u^{\prime}(n)$ (here primed indices are used to distinguish between the two different subalgebras).

\section{3 $N=1$ SUSY}

As shown in sect. 9, there exist $N=1$ supersymmetric truncations of the system (5.1)(5.5), based on antiautomorphisms. To study which of finite-dimensional global symmetries survive in this case, let us turn to the truncation conditions (9.5) based on the symmetry (9.15) in the gauge field sector, starting with the Abelian internal symmetry. Using the definition of the antiautomorphism $\sigma$ (4.21), one finds that this truncation eliminates the generators $Q_{\alpha}^{(2)}$ and $J$ from the set of $\operatorname{osp}(2,2)$ generators (10.4), while the generators $T_{\alpha \beta}$ and $Q_{\alpha}^{(1)}$ survive. Thereby, this truncation breaks $N=2$ SUSY down to $N=1$ SUSY $\operatorname{osp}(1,2) \oplus \operatorname{osp}(1,2)$ with the generators

$$
T_{ \pm, \alpha \beta}=\frac{1}{4 i} \Pi_{ \pm}\left\{\tilde{y}_{\alpha}, \tilde{y}_{\beta}\right\}, \quad Q_{ \pm, \alpha}=\Pi_{ \pm} \tilde{y}_{\alpha}
$$

The conditions (9.5), (9.16) eliminate from the expansion (10.5) the $\psi_{1}$-dependent components of the scalars $C_{ \pm}$and the fermionic components that can depend on $k$ and $\psi_{1}$ only via $k \psi_{1}$. As a result, the truncated $N=1$ matter supermultiplet contains 2 scalars and 2 spinors,

$$
\left\{C_{+}^{0}(x), C_{-}^{0}(x), C_{1 \alpha}^{0}(x), C_{0 \alpha}^{1}(x)\right\}
$$

(with the convention $C(k)=C_{0}+C_{1} k$ ) with the masses (3.22) and (3.23) for bosons and fermions respectively.

The extended systems (5.1)-(5.5) in this case have the global symmetries $h o_{\beta}(n, m \mid 2)$ and $\operatorname{husp}_{\beta}(n, m \mid 2)$ with the orthogonal and symplectic algebras in the Yang-Mills sector. At $\beta=1$ and $n=m$, the maximal finite-dimensional global symmetry subalgebras are $\operatorname{osp}(1,2) \oplus \operatorname{osp}(1,2) \oplus o(n) \oplus o(n)$ or $\operatorname{osp}(1,2) \oplus \operatorname{osp}(1,2) \oplus u s p(n) \oplus u s p(n)$, depending on a sign in (9.14) (the doubling is again due to $\left.\psi_{1}\right)$. At $\beta=0(\alpha=1)$ and arbitrary $n, m$, the maximal finite-dimensional subalgebra is either $\operatorname{osp}(1,2) \oplus o s p(1,2) \oplus o(n) \oplus o(m)$ or $\operatorname{osp}(1,2) \oplus \operatorname{osp}(1,2) \oplus u s p(n) \oplus u s p(m)$.

To find out what $N=1$ matter multiplets survive in the non-Abelian case one should analyze the truncation conditions (9.5), (9.16) in the sector of matter fields. Let us start with the case $\alpha=0, \beta=1$, and $n=m$, which corresponds to the internal symmetries with diagonal $o(n) \oplus o(n)$ and $u s p(n) \oplus u s p(n)$. The dynamical matter fields $C$ (10.5) are arranged into $2 n \times 2 n$ matrix

$$
\left(\begin{array}{cc}
C_{1} & C_{1 \alpha} \\
C_{2 \alpha} & C_{2}
\end{array}\right),
$$

with bosons in the diagonal $n \times n$ blocks $C_{1}, C_{2}$, and fermions in the off-diagonal $n \times$ $n$ blocks $C_{1 \alpha}, C_{2 \alpha}$. All components depend on $\psi_{1}$ and are proportional to $\psi_{2}$. The dependence on $k$ is eliminated by the projection condition (9.11). Then, the truncation condition for the dynamical scalar fields $C^{\text {diag }}\left(\psi_{1}\right)=C_{1}\left(\psi_{1}\right) \oplus C_{2}\left(\psi_{1}\right)$ reads

$$
H C^{\text {diag }}\left(\psi_{1}\right) H^{-1}=\left(C^{\text {diag }}\left(-\psi_{1}\right)\right)^{T} .
$$


In terms of matrix elements of the diagonal blocks this yields taking into account (9.14)

$H^{i j} C_{j}^{k}\left(\psi_{1}\right)=H^{l k} C_{l}^{i}\left(-\psi_{1}\right)= \pm H^{k l} C_{l}^{i}\left(-\psi_{1}\right), \quad i, j=1, \ldots, n \quad$ or $\quad i, j=n+1, \ldots, 2 n$.

Denoting $\left(C^{\text {diag }}\right)^{i k}=H^{i j}\left(C^{\text {diag }}\right)_{j}{ }^{k}$ we get $\left(C^{\text {diag }}\left(\psi_{1}\right)\right)^{i k}= \pm\left(C^{\text {diag }}\left(-\psi_{1}\right)\right)^{k i}$, i.e. the components singled out by the projectors (10.3) turn out to be related to each other.

Taking into account that all dynamical fields are proportional to $\psi_{2}$ and that the components in the direct sums $o(n) \oplus o^{\prime}(n)$ and $u s p(n) \oplus u s p^{\prime}(n)$ are singled out by the projector (10.3), one concludes that the physical bosons in each of the diagonal blocks in (10.9) belong to the representation $n \otimes n^{\prime}$, so that the full set of the independent bosonic fields belongs to the representation $2\left(n \otimes n^{\prime}\right)$ of the internal symmetry. Analogous conditions for the dynamical spinors $C_{\alpha}\left(\psi_{1}\right)=C_{1 \alpha}\left(\psi_{1}\right) \oplus C_{2 \alpha}\left(\psi_{1}\right)$ read

$$
C_{\alpha}^{i^{\prime} k^{\prime \prime}}\left(\psi_{1}\right)=\mp C_{\alpha}^{k^{\prime \prime} i^{\prime}}\left(-\psi_{1}\right), \quad i^{\prime}=1, \ldots, n, \quad k^{\prime \prime}=n+1, \ldots, 2 n .
$$

As a result we find that the fermions also belong to the representation $2\left(n \otimes n^{\prime}\right)$.

In the case $\alpha=1(\beta=1, n=m)$ the similar analysis (with the symmetry properties of bosons and fermions interchanged) leads to the same result with the matter fields in the representation $2\left(n \otimes n^{\prime}\right)$ of the internal symmetries $o(n) \oplus o^{\prime}(n)$ and $u s p(n) \oplus u s p^{\prime}(n)$.

The case $\alpha=1, \beta=0$ with arbitrary $n$ and $m$ can be considered analogously and corresponds to the representations $2(n \otimes m)$ of the internal symmetries $o(n) \oplus o(m)$ and $u s p(n) \oplus u s p(m)$.

\subsection{Non-supersymmetric Case $(N=0)$}

The system (5.1)-(5.5) with the global symmetries $h u_{\beta}(n, m \mid 2), h o_{\beta}(n, m \mid 2)$, and $h_{u s p}(n, m \mid 2)$ at $\beta=1$ and $n \neq m$ do not possess global supersymmetries. In this case one can consider only the $o(2,2) \oplus o(2,2)$ multiplets in representations of inner symmetries.

In the systems based on $h u_{01}(n, m \mid 4)$ with arbitrary $n$ and $m$, bosons belong to the representation $\left(n \otimes \bar{n}^{\prime}\right) \oplus\left(m \otimes \bar{m}^{\prime}\right) \oplus\left(\bar{n} \otimes n^{\prime}\right) \oplus\left(\bar{m} \otimes m^{\prime}\right)$, while fermions belong to the representation $\left(n \otimes \bar{m}^{\prime}\right) \oplus\left(m \otimes \bar{n}^{\prime}\right) \oplus\left(\bar{m} \otimes n^{\prime}\right) \oplus\left(\bar{n} \otimes m^{\prime}\right)$ of the internal symmetry $u(n) \oplus u(m) \oplus u^{\prime}(n) \oplus u^{\prime}(m)$.

In the systems based on $h o_{01}(n, m \mid 4)$ and $h u s p_{01}(n, m \mid 4)$ bosons belong to the representation $\left(n \otimes n^{\prime}\right) \oplus\left(m \otimes m^{\prime}\right)$, while fermions belong to the representation $\left(n \otimes m^{\prime}\right) \oplus\left(m \otimes n^{\prime}\right)$ of the internal symmetries $o(n) \oplus o(m) \oplus o^{\prime}(n) \oplus o^{\prime}(m)$ or $u s p(n) \oplus u s p(m) \oplus u s p^{\prime}(n) \oplus u s p^{\prime}(m)$.

In the case $\alpha=1$ and $\beta=1$, representations are of the same structure, but with the symmetry properties of bosons and fermions interchanged.

Analogously, at $\beta=0(\alpha=1)$ one can consider the $o(2,2) \oplus o(2,2)$ multiplets in representations of broader inner symmetries, if one does not require them to commute with the SUSY subalgebras. Then, in the systems based on $h u_{10}(n, m \mid 4)$ bosons belong to the representation $(n \otimes \bar{m}) \oplus(m \otimes \bar{n}) \oplus\left(n^{\prime} \otimes \bar{m}^{\prime}\right) \oplus\left(m^{\prime} \otimes \bar{n}^{\prime}\right)$, while fermions belong to the representation $\left(n \otimes \bar{m}^{\prime}\right) \oplus\left(m \otimes \bar{n}^{\prime}\right) \oplus\left(\bar{m} \otimes n^{\prime}\right) \oplus\left(\bar{n} \otimes m^{\prime}\right)$ of the internal symmetry $u(n) \oplus$ $u(m) \oplus u^{\prime}(n) \oplus u^{\prime}(m)$. This is a manifestation of the important fact that the same infinitedimensional HS algebras can have different maximal finite-dimensional subalgebras which contain the space-time (AdS) symmetry algebra as a subalgebra. 
In the systems based on $h o_{10}(n, m \mid 4)$ and $h u s p_{10}(n, m \mid 4)$ bosons belong to the representation $(n \otimes m) \oplus\left(n^{\prime} \otimes m^{\prime}\right)$, while fermions belong to the representation $\left(n \otimes m^{\prime}\right) \oplus\left(m \otimes n^{\prime}\right)$ of the internal symmetries $o(n) \oplus o(m) \oplus o^{\prime}(n) \oplus o^{\prime}(m)$ and $u s p(n) \oplus u s p(m) \oplus u s p^{\prime}(n) \oplus u s p^{\prime}(m)$.

Finally, in the non-supersymmetric case it is of course possible to truncate out fermions completely by imposing the condition $f\left(A_{i}\right)=A_{i}, A_{i}=\left(W_{\mu}, B, S_{\alpha}\right)$, with $f(9.6)$.

\subsection{Massless Case and N-Extended SUSY}

It turns out that in the massless case $\nu=0$ the system (5.1)-(5.5) admits an additional truncation based on the automorphism $k \rightarrow-k$. The corresponding involutive symmetry $\zeta$

$$
\zeta[W(k)]=W(-k), \quad \zeta\left[S_{\alpha}(k)\right]=S_{\alpha}(-k), \quad \zeta[B(k)]=-B(-k),
$$

induces in the $\beta=0$ case the truncation conditions $\zeta\left(A_{i}\right)=A_{i}, A_{i}=\left(W_{\mu}, B, S_{\alpha}\right)$. It is important that at $\nu=0$ the vacuum solutions (6.1), (6.6), and (6.14) are compatible with these conditions.

In the non-Abelian case with $\beta=1$ and $n=m$, one should consider the automorphism $k \rightarrow-k$ combined with some involutive automorphism $\chi, \chi(U)=-U$, to ensure that the projection conditions (9.11) are invariant. One can define $\chi$ on $M_{a t} t_{2 n}$ as

$$
\chi(M)=Y M Y^{-1}, \quad Y=\left(\begin{array}{cc}
0 & I_{n} \\
I_{n} & 0
\end{array}\right) .
$$

As a result, a generalization of the truncation induced by (10.12) to all supersymmetric cases is the truncation by $\zeta \chi^{\beta}$.

The truncation based on $\zeta$ reduces our system to that proposed in [41]. The reduced system possesses $N=1$ SUSY $\operatorname{osp}(1,2) \oplus \operatorname{osp}(1,2)$ with the generators (10.7). The set of fields obtained from (10.6) with the aid of this truncation turns out to be reducible. One can truncate the system further with the aid of the symmetry (9.15)-(9.17) which preserves $N=1 \mathrm{SUSY}$. As a result, in the Abelian case we arrive at the following $N=1$ massless supermultiplet

$$
\left\{C_{1}^{0}(x), C_{1 \alpha}^{0}(x)\right\} .
$$

In the non-Abelian case, we arrive at the massless $N=1$ supermultiplets in the representations $n \otimes n^{\prime}(\beta=1, n=m)$ or $n \otimes m(\beta=0, \alpha=1)$ of the corresponding internal symmetries of subsect. 10.3. (Note that the non-Abelian truncation based on the symmetry $\zeta \chi^{\beta}$ does not affect the inner symmetries.)

In the massless case $\nu=0$ there exists an interesting alternative truncation based on the symmetry (10.12), which preserves $N=2$ SUSY. Consider the combination $\zeta \chi^{\beta} \eta$ of the symmetries (10.12), (10.13) and the symmetry (9.15)-(9.17) based on the antiautomorphism $\sigma$ of subsect. 9.3. In the Abelian case, the corresponding truncation conditions reduce the $N=2$ hypermultiplet (10.6) to the following $N=2$ massless supermultiplet,

$$
\left\{C_{1}^{0}(x), C_{0}^{1}(x), C_{0 \alpha}^{0}(x), C_{1 \alpha}^{0}(x)\right\} .
$$

In the non-Abelian case, we arrive at the $N=2$ massless supermultiplet either in the representations $2\left(n \otimes n^{\prime}\right)$ of the internal symmetries $o(n) \oplus o^{\prime}(n)$ and $u s p(n) \oplus u s p^{\prime}(n)$ 
$(\beta=1, n=m)$, or in the representations $2(n \otimes m)$ of $o(n) \oplus o(m)$ and $u s p(n) \oplus u s p(m)$ $(\beta=0$, arbitrary $n, m)$. This additional reduction compatible with $N=2$ SUSY is a manifestation of the well-known shortening of massless supermultiplets.

Finally let us discuss a possibility to have extended supersymmetry with $N>2$. In [27], it was shown that there is a simple way to incorporate $N$-extended superalgebras $\operatorname{osp}(N, 2 m)$ ( $m=1$ for the $d=3$ case under consideration) by supplementing the bosonic generating elements of the Heisenberg algebra $y_{\alpha}$ with the Clifford elements $\phi^{i}(i=$ $1, \ldots, N)$. In this approach the Clifford algebra $C_{N}$ is a particular case of the matrix

algebra $\operatorname{Mat}_{2^{\frac{N}{2}}}$ (for $N$ even), while the generators of the $\operatorname{ssp}(N, 2 m)$ are realized in terms of the bilinears

$$
T_{\alpha \beta}=\left\{y_{\alpha}, y_{\beta}\right\}, \quad Q_{\alpha}^{i}=y_{\alpha} \phi^{i}, \quad M^{i j}=\left[\phi^{i}, \phi^{j}\right],
$$

provided that

$$
\left[y_{\alpha}, y_{\beta}\right]=2 i \epsilon_{\alpha \beta}, \quad\left\{\phi^{i}, \phi^{j}\right\}=2 \delta^{i j} .
$$

It is this realization of the $d 4 N=8$ extended supersymmetry which was recently used in [25] to discuss the $N=8$ version of the $d=4$ HS model.

Now we observe that this construction is not working for the deformed oscillators because the generators (10.16) do not form a closed algebra if $y_{\alpha}$ is replaced by $\hat{y}_{\alpha}$ (3.1) with $\nu \neq 0$. This result can be explained as follows. For $\nu \neq 0$ the mass of the matter supermultiplet is non-vanishing and the spin range within a supermultiplet increases with $N$. Since massive fields of spins greater than $1 / 2$ are not included in our model, $N>2$ extended supersymmetry cannot be realized. In the massless case, however, one can realize higher supersymmetries within only scalar and spinor fields 662 due to trivialization of the notion of spin for the $d=3$ massless case (i.e. trivialization of the $d=3$ massless little group).

Thus we conclude that the model under consideration admits $N>2$ extended supersymmetry only for the massless vacuum $\nu=0$. The conclusion that arbitrary high $N$ is allowed differs from the conclusions of [62] because our model is more general due to the presence of the HS gauge interactions. It is worth to mention that $d=3$ massless fields can be interpreted [63] as $d=4$ singletons.

\section{Conclusion}

The $d 3$ model analyzed in this paper is shown to describe HS gauge interactions of massive $N=2$ hypermultiplets. The parameter of mass of the matter fields appears as a vacuum expectation value of a certain auxiliary scalar field. The model admits a generalization with classical Yang-Mills groups which are of the unitary type for the $N=2$ supersymmetric case and either orthogonal or symplectic for the $N=1$ reductions, in striking parallelism with superstring theory. Moreover, similarly to the previously obtained results for $d 4$ HS models [58, 28], the $N=1$ reductions are obtained with the aid of a certain antiautomorphism of the Heisenberg-Weyl algebra, which is a counterpart of the twist (orientation) operator in string theory.

An important result of the paper, which is expected to have implications for the theory of HS gauge fields in various dimensions (and, hopefully, also for its lower-spin reductions 
like supergravity), is that the proposed dynamical equations admit an integrating flow which relates solutions of the non-linear system to those of the linearized system and is in many respects similar to the Bäcklund transformations in integrable systems and to the Nicolai map [42] in supersymmetric models. Apart from the fact that this integrating flow allows one to build constructively perturbative solutions of the model, it raises an important question of the proper definition of the concept of locality in the models with a dimensionful parameter like a cosmological constant, which we hope to discuss in a future publication. As we demonstrate in this paper, a similar phenomenon takes place in ordinary $d 3$ gravity with the cosmological term, where it is possible to perform a weakly local (i.e. containing non-localities that can be expanded in infinite series of higher derivatives) field redefinition which compensates the stress energy tensor on the right hand side of the equations of motion thus reducing the problem to the vacuum case with zero-curvature equations. An important difference, however, is that in the case of the HS models we are able to write down in a very simple and explicit form the integrating flow that governs such a field redefinition in all orders in interactions. A related interesting question consists in the generalization of the cohomological analysis of the stress-energy tensor, performed in Appendix $\mathrm{C}$ of this paper, to all conserved HS currents. One can expect that, similarly to the case of gravity, all HS currents belong to the trivial cohomology class with respect to the weakly local transformations, but have to belong to a non-trivial cohomology class with respect to local transformations.

Another interesting related topic is a proper definition of locality in the $d 4$ HS gauge theories. In the context of the results of this paper one can speculate that the fact that $d 4 \mathrm{HS}$ gauge theories require non-zero cosmological constant 14 might be a signal of some sort of non-locality of the HS gauge theories beyond the cubic order (in the cubic order the number of derivatives of some field of a given spin $s$ in the interaction part of the action is bounded by $s$ [14). This hypothesis is very interesting in the context of the applications to $M$ theory.

An intriguing problem for the future is to analyze in detail what happens at the special points $\nu=2 l+1, l \in \mathbf{Z}$. In [44], it is shown that at these points the free field equations effectively start from the some higher Lorentz multispinors rather than from a scalar. The same singular points appear in the vacuum solution (6.6) (see also Appendix B). Remarkably, the values $\nu=1,3$ correspond to the case of $d 3$ Maxwell electrodynamics [44]. The physical meaning of the higher singular values of $\nu$ is not yet clear. The mathematical interpretation is very simple however. These special values correspond to all those values of the Casimir operators of the AdS algebra $o(2,2) \sim s p(2) \oplus s p(2)$ which correspond to its finite-dimensional representations. In other words, the situation becomes special when the relevant representations of the AdS algebra admit singular vectors while the infinite-dimensional HS algebra acquires ideals.

\section{Acknowledgments}

Authors are grateful to R. R. Metsaev for a useful comment. This research was supported in part by INTAS, Grants No.96-0538, No.96-0308, and by the RFBR Grant No.96-0101144. S. P. acknowledges a partial support from the Landau Scholarship Foundation, 
Forschungszentrum Jülich.

\section{Appendix A. Regularity}

In this appendix, we define a class of functions in the auxiliary spinor spaces, used throughout the paper. This regularity class generalizes that introduced in [53].

Definition. A function $f\left(z, y ; k, \psi_{1,2}\right)$ is called regular if it can be expanded into a finite sum of some functions $g$ of the form

$$
g\left(z, y ; k, \psi_{1,2}\right)=P\left(z, y ; k, \psi_{1,2}\right) \int_{M^{n}} d^{n} t \rho(t) \exp [i \phi(t)(z y)],
$$

where the integration is carried out over some compact domain $M^{n} \subset R^{n}$ with the coordinates $t_{i}(i=1, \ldots, n), P\left(z, y ; k, \psi_{1,2}\right)$ is an arbitrary polynomial of $z, y, k$ and $\psi_{1,2}, \phi(t)$ is an arbitrary polynomial function of $t_{i}$, while $\rho(t)$ is some absolutely integrable function on $M^{n}$.

Comment 1. This definition contains individual exponentials with arbitrary polynomial pre-exponential factors via formulae like $\exp a(z y)=\int_{0}^{1} d t \frac{\partial}{\partial t}[t \exp [t a(z y)]]$. Equivalently one can allow $\rho(t)$ to contain an arbitrary finite number of $\delta$-functions and their derivatives.

Theorem. Given regular functions $g_{1}\left(z, y ; k, \psi_{1,2}\right)$ and $g_{2}\left(z, y ; k, \psi_{1,2}\right)$, their product (4.1) $\left(g_{1} * g_{2}\right)\left(z, y ; k, \psi_{1,2}\right)$ is some regular function.

Proof.

$$
\begin{gathered}
g_{1} * g_{2}=P_{1}(z, y) \int_{M_{1}} d t_{1} \rho_{1}\left(t_{1}\right) \exp \left[i \phi_{1}\left(t_{1}\right)(z y)\right] * P_{2}(z, y) \int_{M_{2}} d t_{2} \rho_{2}\left(t_{2}\right) \exp \left[i \phi_{2}\left(t_{2}\right)(z y)\right]= \\
=\int_{M_{1}} d t_{1} \rho_{1}\left(t_{1}\right) \exp \left[i \phi_{1}\left(t_{1}\right)(z y)\right] \int_{M_{2}} d t_{2} \rho_{2}\left(t_{2}\right) \exp \left[i \phi_{2}\left(t_{2}\right)(z y)\right] \times \\
\times \frac{1}{(2 \pi)^{2}} \iint d u d v \exp \left\{i(u v)+i \phi_{1}\left(t_{1}\right)[(z-y) u]+i \phi_{2}\left(t_{2}\right)[(z+y) v]\right\} P_{1}(z+u, y+u) P_{2}(z-v, y+v) .
\end{gathered}
$$

Inserting

$$
P(z+a, y+b)=\left.\exp \left[a^{\alpha} \frac{\partial}{\partial z_{1}^{\alpha}}+b^{\alpha} \frac{\partial}{\partial y_{1}^{\alpha}}\right] P\left(z_{1}, y_{1}\right)\right|_{\substack{z_{1}=z \\ y_{1}=y}}
$$

one gets

$$
\begin{gathered}
g_{1} * g_{2}=\int_{M_{1} \times M_{2}} d t_{1} d t_{2} \rho_{1}\left(t_{1}\right) \rho_{2}\left(t_{2}\right) \exp \left\{i\left[\phi_{1}\left(t_{1}\right)+\phi_{2}\left(t_{2}\right)\right](z y)\right\} \times \\
\times\left.\frac{1}{(2 \pi)^{2}} \iint d u d v \exp \left\{u^{\alpha}\left[i \phi_{1}\left(t_{1}\right)(z-y)_{\alpha}+\frac{\partial}{\partial z_{1}^{\alpha}}+\frac{\partial}{\partial y_{1}^{\alpha}}\right]\right\} P_{1}\left(z_{1}, y_{1}\right)\right|_{\substack{z_{1}=z \\
y_{1}=y}} \times \\
\times\left.\exp \left\{i v^{\alpha}\left[\phi_{2}\left(t_{2}\right)(z+y)_{\alpha}+u_{\alpha}-i\left(\frac{\partial}{\partial y_{2}^{\alpha}}-\frac{\partial}{\partial z_{2}^{\alpha}}\right)\right]\right\} P_{2}\left(z_{2}, y_{2}\right)\right|_{\substack{z_{2}=z \\
y_{2}=y}}=
\end{gathered}
$$




$$
\begin{gathered}
=\int_{M_{1} \times M_{2}} d t_{1} d t_{2} \rho_{1}\left(t_{1}\right) \rho_{2}\left(t_{2}\right) \exp \left\{i\left[\phi_{1}\left(t_{1}\right)+\phi_{2}\left(t_{2}\right)-2 \phi_{1}\left(t_{1}\right) \phi_{2}\left(t_{2}\right)\right](z y)\right\} \times \\
\times \exp \left\{-i \epsilon^{\alpha \beta}\left(\frac{\partial}{\partial z_{1}^{\alpha}}+\frac{\partial}{\partial y_{1}^{\alpha}}\right)\left(\frac{\partial}{\partial z_{2}^{\beta}}-\frac{\partial}{\partial y_{2}^{\beta}}\right)\right\} P_{1}\left[z_{1}-\phi_{2}\left(t_{2}\right)(z+y), y_{1}-\phi_{2}\left(t_{2}\right)(z+y)\right] \times \\
\times\left. P_{2}\left[z_{2}-\phi_{1}\left(t_{1}\right)(z-y), y_{2}+\phi_{1}\left(t_{1}\right)(z-y)\right]\right|_{\substack{z_{1}=z_{2}=z \\
y_{1}=y_{2}=y}} .
\end{gathered}
$$

Since the product of the two compact domains $M_{1} \subset R_{n}$ and $M_{2} \subset R_{m}$ is a compact domain in $R_{n+m}$ and $P_{1}, P_{2}$ are some polynomials, one concludes that the latter expression is a finite sum of some regular functions (A.1).

Comment 2. In this proof it is important that the determinant of the quadratic form in the Gaussian integral is a constant independent of the particular choice of the functions $g_{1}$ and $g_{2}$. This property is a consequence of the fact that we use the star product (4.1) and might not be true for other star-product formulae. As an example, the reader can check that $\exp [i(z y)] * \exp [i(z y)]$ does not exist for the star product corresponding to the Weyl ordering.

From the theorem above it follows that every finite-order perturbative computation that starts with some regular generating functions $W, B$, and $S$ is well defined. In particular the vacuum solutions $S_{0 \alpha}$ and $\tilde{y}_{\alpha}$ belong to the regularity class.

\section{Appendix B. Construction of Vacuum Solutions}

The problem is to find elements $\tilde{y}_{\alpha}$ and $S_{0 \alpha}$ such that

$$
\begin{gathered}
{\left[\tilde{y}_{\alpha}, \tilde{y}_{\beta}\right]_{*}=2 i \epsilon_{\alpha \beta}(1+\nu k), \quad \tilde{y}_{\alpha} k=-k \tilde{y}_{\alpha},} \\
{\left[S_{0 \alpha}, S_{0 \beta}\right]_{*}=-2 i \epsilon_{\alpha \beta}(1+\nu K), \quad S_{0 \alpha} * K=-K * S_{0 \alpha},} \\
{\left[S_{0 \alpha}, \tilde{y}_{\beta}\right]_{*}=0 .}
\end{gathered}
$$

Let us start with $\tilde{y}_{\alpha}$. A Lorentz covariant Ansatz is

$$
\tilde{y}_{\alpha}=\int_{-1}^{1} d s\left[\left(y_{\alpha}+z_{\alpha}\right) n(s, k)+\left(y_{\alpha}-z_{\alpha}\right) m(s, k)\right] \exp \left[\frac{i}{2}(s+1)\left(z_{\alpha} y^{\alpha}\right)\right] \text {. }
$$

A direct computation shows that

$$
\begin{aligned}
\tilde{y}_{\alpha} * \tilde{y}^{\alpha} & =\int d s d s^{\prime} \theta(1+s) \theta(1-s) \theta\left(1+s^{\prime}\right) \theta\left(1-s^{\prime}\right) \exp \left[\frac{i}{2}\left(1-s s^{\prime}\right)\left(z_{\alpha} y^{\alpha}\right)\right] \\
& \times\left[\left(8 i+2 s s^{\prime}\left(z_{\alpha} y^{\alpha}\right)\right) n(s,-k) m\left(s^{\prime}, k\right)-2\left(z_{\alpha} y^{\alpha}\right) m(s,-k) n\left(s^{\prime}, k\right)\right] .
\end{aligned}
$$

Let us require the following condition to be true,

$$
\begin{gathered}
\int d s d s^{\prime} \theta(1+s) \theta(1-s) \theta\left(1+s^{\prime}\right) \theta\left(1-s^{\prime}\right) \delta\left(t-s s^{\prime}\right) n(s,-k) m\left(s^{\prime}, k\right) \\
=\frac{1}{8}(1+t)[\delta(t-1)+\delta(t+1)+\nu k] .
\end{gathered}
$$


Inserting this into (B.5) one finds that

$$
\tilde{y}_{\alpha} * \tilde{y}^{\alpha}=2 i(1+\nu k)
$$

which is an equivalent form of (B.1).

Thus, every solution of (B.6) gives rise to some $\tilde{y}_{\alpha}$ (B.1). Let us now construct a class of solutions of $(\mathrm{B} .6)$ with the aid of the following Ansatz

$$
n(s, k)=\frac{1}{2}(1+\gamma s) p(s, k), \quad m(s, k)=\frac{1}{2}(1+\gamma s) q(s, k)
$$

with

$$
\gamma= \pm 1, \quad p(s, k)=p(-s, k), \quad q(s, k)=q(-s, k) .
$$

These symmetry properties prove that

$$
\int_{-1}^{1} d s \int_{-1}^{1} d s^{\prime} \delta\left(t-s s^{\prime}\right)\left(s+s^{\prime}\right) p(s) q\left(s^{\prime}\right)=0 .
$$

As a result (B.6) reduces to the form

$$
(p(-k) \circ q(k))(t)=\frac{1}{2}[\delta(t-1)+\delta(t+1)+\nu k],
$$

where a new product $\circ$ is defined by the relation

$$
(p \circ q)(t)=\int d s d s^{\prime} \theta(1+s) \theta(1-s) \theta\left(1+s^{\prime}\right) \theta\left(1-s^{\prime}\right) \delta\left(t-s s^{\prime}\right) p(s) q\left(s^{\prime}\right) \text {. }
$$

It is easy to see that this product law is commutative and associative.

To solve (B.11) it is convenient to use the following trick. Consider the expansion

$$
p(t)=\sum_{n=0}^{\infty} p_{n} I_{n}(t)
$$

where the basis functions $I_{n}$ are defined by the multiple integrals

$$
I_{n}(t)=\int d^{n} s \theta\left(1+s_{1}\right) \ldots \theta\left(1+s_{n}\right) \theta\left(1-s_{1}\right) \ldots \theta\left(1-s_{n}\right) \delta\left(t-s_{1} s_{2} \ldots s_{n}\right)
$$

for $n>0$, and

$$
I_{0}=\frac{1}{2}[\delta(t-1)+\delta(t+1)]
$$

It is easy to evaluate the basis integrals $I_{n}$,

$$
I_{n}=\frac{\left(-\ln t^{2}\right)^{n-1}}{(n-1) !}, \quad n>0 .
$$

One can check that so defined functions $I_{n}$ have the following basic property,

$$
I_{n} \circ I_{m}=I_{n+m} \quad \forall n \geq 0 .
$$


Therefore, given $p(s)(\overline{\mathrm{B} .13})$ we can define its symbol $\tilde{p}(x)$,

$$
\tilde{p}(x)=\sum_{n=0}^{\infty} p_{n} x^{n}
$$

an ordinary function of the variable $x$. From (B.17) it follows then that

$$
(\widetilde{p \circ q})(x)=\tilde{p}(x) \tilde{q}(x) \text {. }
$$

The equation (B.11) rewritten in terms of symbols has a very simple form

$$
\tilde{p}(x,-k) \tilde{q}(x, k)=1+\frac{1}{2} \nu k x
$$

and admits a class of solutions parametrized by an arbitrary function. This ambiguity takes its origin in the gauge ambiguity (7.11). A solution of particular interest is the one that transforms properly under the involution (4.20) and the antiautomorphism (4.21), $\sigma\left(\tilde{y}_{\alpha}\right)=i \tilde{y}_{\alpha}$ and $\tilde{y}_{\alpha}^{\dagger}=\tilde{y}_{\alpha}$. This is the case provided that

$$
n(s,-k)=m(s, k), \quad \bar{n}(s, k)=n(s, k), \quad \bar{m}(s, k)=m(s, k) .
$$

According to $(\overline{\mathrm{B} .8})$, this implies that $p$ and $q$ are real and

$$
p(s,-k)=q(s, k) .
$$

Now we see that there is only one (real) solution of (B.20), satisfying (B.22),

$$
\tilde{q}_{s y m}(x, k)=\sqrt{1+\frac{1}{2} \nu k x}, \quad \tilde{p}_{\text {sym }}(x, k)=\sqrt{1-\frac{1}{2} \nu k x} .
$$

Thus, the symmetry requirement selects a vacuum solution uniquely. Relaxing this symmetry requirement one can find many solutions. The simplest ones are

$$
\tilde{q}=1, \quad \tilde{p}=1-\frac{1}{2} \nu k x
$$

or

$$
\tilde{q}=1+\frac{1}{2} \nu k x, \quad \tilde{p}=1 .
$$

One can consider a one-parametric interpolating class of solutions $\left\{\tilde{y}_{\alpha}^{\tau}\right\}$,

$$
\tilde{q}_{\tau}(x, k)=\left(1+\frac{1}{2} \nu k x\right)^{\tau}, \quad \tilde{p}_{\tau}(x, k)=\left(1-\frac{1}{2} \nu k x\right)^{1-\tau}
$$

Once the symbols $\tilde{p}$ and $\tilde{q}$ are known, one can compute the expansion coefficients in $(\mathbb{B} .18)$ and then reconstruct the original functions $p(s, k)$ and $q(s, k)$ via $(\mathbb{B . 1 3})$, (B.15), and $(\mathbb{B} .16)$. In what follows, we will set $\gamma=-1$ to have solutions that start with $y_{\alpha}$ in the zero order in $\nu$ 四.

\footnotetext{
${ }^{19}$ In the case $\gamma=1$ a solution contains an additional exponential factor, but it can be shown to be equivalent to that with $\gamma=-1$ by an automorphism.
} 
The polynomial solutions $(\overline{\mathrm{B} .24}),(\overline{\mathrm{B} .25})$, which correspond to $\tau=0$ and $\tau=1$, give rise, respectively, to the solutions $\tilde{y}_{\alpha}^{+}$and $\tilde{y}_{\alpha}^{-}$(6.10). The symmetric solution (B.23) corresponds to $\tau=\frac{1}{2}$ and gives rise to $\tilde{y}_{\alpha}^{\text {sym }}(6.11)$.

Expanding (B.26) in power series and using (B.15) and (B.16) one finds

$$
q_{\tau}(s, k)=\frac{1}{2}\left[\delta(s-1)+\delta(s+1)+k \nu \tau \Phi\left(1-\tau, 2 ; \ln |s|^{\nu k}\right)\right]
$$

and

$$
p_{\tau}(s, k)=\frac{1}{2}\left[\delta(s-1)+\delta(s+1)-k \nu(1-\tau) \Phi\left(\tau, 2 ;-\ln |s|^{\nu k}\right)\right] .
$$

Here $\Phi(a, c ; x)$ is the degenerate hypergeometric function defined by the power series

$$
\Phi(a, c ; x)=1+\frac{a x}{c 1 !}+\frac{a(a+1) x^{2}}{c(c+1) 2 !}+\ldots
$$

At $0<a<c$ it admits a useful integral representation 64

$$
\Phi(a, c ; x)=\frac{\Gamma(c)}{\Gamma(a) \Gamma(c-a)} \int_{0}^{1} d t e^{t x} t^{a-1}(1-t)^{c-a-1},
$$

which leads to

$$
\begin{aligned}
& q_{\tau}(s, k)=\frac{1}{2}\left[\delta(s-1)+\delta(s+1)+k \frac{\nu \tau}{\Gamma(1-\tau) \Gamma(1+\tau)} \int_{0}^{1} d t|s|^{t \nu k} t^{-\tau}(1-t)^{\tau}\right], \\
& p_{\tau}(s, k)=\frac{1}{2}\left[\delta(s-1)+\delta(s+1)-k \frac{\nu(1-\tau)}{\Gamma(\tau) \Gamma(2-\tau)} \int_{0}^{1} d t|s|^{-t \nu k} t^{\tau-1}(1-t)^{1-\tau}\right],
\end{aligned}
$$

with $0<\tau<1$.

From these formulae we see that the corresponding $\tilde{y}_{\alpha}(\mathbb{B} .4)$ is well defined at least in some neighborhood of $\nu=0$. The case of $\tau=\frac{1}{2}$ is most interesting. It is not difficult to see that the solution $\tilde{y}^{\text {sym }}$ makes sense for $\nu<1$ while some singularity can appear at $\nu=1$. This is just the closest to zero special value of $\nu$ discussed in sect. 3. In fact, we expect that our results can be extended to arbitrary values of $\nu$ with some specificities for $\nu=2 l+1, l \in \mathbf{Z}$. To achieve this one has to analyze the problem more accurately at the singular point $t=0$ in $(\mathbb{B} .16)$. We hope to discuss this intriguing issue elsewhere.

To find $S_{0 \alpha}$ satisfying (B.2), one can use the same Ansatz (B.4) with the substitutions $k \rightarrow K=k e^{i\left(z_{\alpha} y^{\alpha}\right)}, n(s, k) \rightarrow n(s, k), m(s, k) \rightarrow-m(s, k), y_{\alpha} \rightarrow \rho y_{\alpha}$ and $z_{\alpha} \rightarrow \rho z_{\alpha}$,

$$
\begin{aligned}
S_{0 \alpha}= & \rho \int_{-1}^{1} d s\left\{\left(y_{\alpha}+z_{\alpha}\right) \exp \left[\frac{i}{2}(s+1)\left(z_{\alpha} y^{\alpha}\right)\right] * n(s, K)\right. \\
& \left.-\left(y_{\alpha}-z_{\alpha}\right) \exp \left[\frac{i}{2}(s+1)\left(z_{\alpha} y^{\alpha}\right)\right] * m(s, K)\right\} .
\end{aligned}
$$

Again, (B.2) is a consequence of (B.6).

Further, it can be shown that the elements $\tilde{y}_{\alpha}(\overline{\mathrm{B} .4})$ and $S_{0 \alpha}(\overline{\mathrm{B} .33})$ with the same functions $n(s, k)$ and $m(s, k)$ commute to each other provided that (B.6) is true. To prove this, it is convenient to use the projected expressions $\left[S_{0 \alpha}, \tilde{y}_{\beta}\right]_{*} * \frac{1}{2}\left(1 \pm \exp \left(i z_{\gamma} y^{\gamma}\right)\right)$. 
To summarize, we arrive at the class of the solutions $S_{0 \alpha}^{\tau}$ and $\tilde{y}_{\beta}^{\tau}$ of (B.1)-(B.3). The solutions $\left(S_{0 \alpha}^{+}, \tilde{y}_{\beta}^{+}\right),\left(S_{0 \alpha}^{-}, \tilde{y}_{\beta}^{-}\right)$, and $\left(S_{0 \alpha}^{s y m}, \tilde{y}_{\beta}^{s y m}\right)$ correspond to the cases $\tau=0, \tau=1$, and $\tau=\frac{1}{2}$ respectively.

At the end of this Appendix, let us note that according to Eqs. (B.16), (B.18), the problem of reconstruction of $p(s)$ from its symbol is equivalent to inverting the following remarkable mapping: given $f(x)=\sum_{n=0}^{\infty} f_{n} x^{n}, \tilde{f}=\sum_{n=0}^{\infty} \frac{1}{n !} f_{n} x^{n}$. This mapping has a number of remarkable properties. In particular,

$$
\widetilde{\left(f^{\prime}\right)}=x(\tilde{f})^{\prime \prime}+(\tilde{f})^{\prime}, \quad \widetilde{(x f)}=\int_{0}^{x} d z \tilde{f}(z) .
$$

\section{Appendix C. Example of Gravity}

In this appendix, we use an example of gravity to illustrate how non-local transformations allow one to integrate out non-trivial currents (stress-tensor).

Consider some matter field $C$ interacting with the gravitational field $W^{\alpha \beta}=\omega^{\alpha \beta}+$ $\lambda \psi h^{\alpha \beta}$ expanded near the AdS vacuum solution $W_{0}^{\alpha \beta}=\omega_{0}^{\alpha \beta}+\lambda \psi h_{0}^{\alpha \beta}$ described in sect. 3 . Einstein equations with the cosmological term and a matter source in $2+1$ dimensions can be written in the form

$$
\begin{gathered}
R_{\alpha \beta}=d \omega_{\alpha \beta}-\omega_{\alpha \gamma} \wedge \omega_{\beta}^{\gamma}-\lambda^{2} h_{\alpha \gamma} \wedge h_{\beta}^{\gamma}=\mathcal{T}_{\alpha \beta}(C), \\
r_{\alpha \beta}=d h_{\alpha \beta}-\omega_{\alpha \gamma} \wedge h_{\beta}^{\gamma}-\omega_{\beta \gamma} \wedge h_{\alpha}^{\gamma}=0
\end{gathered}
$$

or, equivalently,

$$
d W_{\alpha \beta}-W_{\alpha \gamma} \wedge W_{\beta}^{\gamma}=\mathcal{T}_{\alpha \beta}(C) .
$$

Here $R_{\alpha \beta}$ and $r_{\alpha \beta}$ are the curvature and torsion 2-forms respectively. The 2-form $\mathcal{T}_{\alpha \beta}(C)$ arises from the energy-momentum tensor $T_{\mu \nu}(C)$ in gravity, when rewriting the Einstein equations $R_{\mu \nu}-\frac{1}{2} g_{\mu \nu} R=T_{\mu \nu}(C)$ (to simplify formulae we discard the coupling constant) in the form (C.1), which is possible in $d=3$. Note that it is assumed in $(\overline{C .3})$ that $\mathcal{T}_{\alpha \beta}(C)$ is independent of the Clifford element $\psi\left(\psi^{2}=1\right)$, which distinguishes between $\omega^{\alpha \beta}$ and $h^{\alpha \beta}$, i.e. the torsion is zero.

Schematically, the mechanism is as follows. Linearized Einstein equations can be cast into the form (with appropriate gauge fixings)

$$
\left(L^{C}-\Lambda^{2}\right) h_{\mu \nu}=T_{\mu \nu}(C)
$$

where $h_{\mu \nu}$ is the fluctuational part of the metric tensor, $L^{C}$ is the linear operator of the equations of the matter fields $L^{C} C=0$, while $\Lambda=\alpha \lambda$ with some numerical coefficient $\alpha \neq 0$ which may depend on a particular choice of the matter sources (e.g. on spin). It is important that when the cosmological constant is non-vanishing, the term with $\Lambda^{2}$ turns out to be non-vanishing too because originally massless fields in the flat space acquire different mass-type terms with the parameter of mass proportional to the inverse AdS radius $m_{s} \sim \gamma_{s} \lambda$. We will call this phenomenon spectra separation. It allows one to solve formally (C.4) by a field redefinition

$$
h_{\mu \nu}^{\prime}=h_{\mu \nu}-\left(L^{C}-\Lambda^{2}\right)^{-1} T_{\mu \nu}(C)=h_{\mu \nu}+\frac{1}{\Lambda^{2}} \sum_{n=0}^{\infty}\left(\frac{1}{\Lambda^{2}} L^{C}\right)^{n} T_{\mu \nu}(C) .
$$


Note that it is important here to use the operator $L^{C}$ to avoid infinite resummations due to collecting similar terms of the type $\left(L^{C}\right)^{n} C\left(L^{C}\right)^{m} C$ which would all be proportional to $C^{2}$ if $L^{C}(C) \sim C \neq 0$. It is also clear that a non-vanishing dimensionful constant, the cosmological constant, plays crucial role in this analysis. A non-local character of the transformation manifests itself in the appearance of infinite series in inverse cosmological constant (equivalently, the radius of the AdS space).

From the equation (C.3) it is obvious that this analysis is cohomological in nature: the form $\mathcal{T}_{\alpha \beta}(C)$ is closed on-mass-shell (this is $d 3$ stress-energy conservation law) but it turns out to be exact, once one relaxes the locality condition.

To make the analysis above more explicit let us consider an example of a massless scalar matter field, using the "unfolded form" (3.20) of its free equations of motion $\square C=\frac{3}{2} \lambda^{2} C$ at $\nu=0$ (with the choice "+"),

$$
D_{0}^{L} C_{\alpha(n)}=\frac{i}{2} \psi\left[h_{0}^{\beta \gamma} C_{\beta \gamma \alpha(n)}-\lambda^{2} n(n-1) h_{0 \alpha \alpha} C_{\alpha(n-2)}\right],
$$

where $D_{0}^{L}$ is the background covariant differential (2.22) and the scalar component $C$ is supposed to be $\psi$-independent. Taking into account $\left(\right.$ C.6) one can write $\mathcal{T}_{\alpha \beta}(C)$ in the form

$$
\mathcal{T}_{\alpha \alpha}(C)=\frac{1}{4} h_{\beta}^{\gamma} \wedge h^{\beta \delta} C_{\gamma \alpha} C_{\delta \alpha}-\frac{1}{2} \mu^{2} h_{\alpha \beta} \wedge h^{\beta}{ }_{\alpha} C^{2},
$$

where $\mu$ is the "AdS mass", $\mu^{2}=-\frac{3}{2} \lambda^{2}$. One can see that $\mathcal{T}_{\alpha \alpha}(C)$ is $\psi$-independent because in accordance with (C.6) $C_{\alpha(n)} \sim \psi^{\frac{n}{2}}$.

The equation (C.3) in the lowest order reads

$$
D_{0} W_{1 \alpha \alpha}=\mathcal{T}_{\alpha \alpha}(C)
$$

where $D_{0}$ is the full background covariant differential,

$$
D_{0} A_{\alpha(n)}=d A_{\alpha(n)}+n W_{0 \alpha}^{\gamma} \wedge A_{\gamma \alpha(n-1)} .
$$

Suppose there exists such a field redefinition

$$
W_{1 \alpha \beta}^{\prime}=W_{1 \alpha \beta}-\psi U_{\alpha \beta}(C)
$$

with some 1-form $U_{\alpha \beta}(C)=U_{\beta \alpha}(C)$, that transforms the equation (C.8) to the vacuum one $D_{0} W_{1 \alpha \alpha}^{\prime}=0$. Obviously, it is possible if

$$
D_{0}\left(\psi U_{\alpha \beta}(C)\right)=\mathcal{T}_{\alpha \beta}(C),
$$

i.e. if $\mathcal{T}_{\alpha \beta}(C)$ is $D_{0}$-exact. Since $\mathcal{T}_{\alpha \beta}(C)$ is $D_{0}$-closed due to (C.8) and the property $D_{0}^{2}=0$, which holds as a consequence of the vacuum equation $R_{0 \alpha \beta}=0$, we arrive at the standard cohomological problem. The fact that the appropriate field redefinition exists implies that $\mathcal{T}_{\alpha \beta}(C)$ belongs to the trivial cohomology class.

To study a question whether $\mathcal{T}_{\alpha \beta}(C)$ is cohomologically trivial in the class of powers series involving arbitrary high derivatives of the matter field $C$ we take into account that 
the multispinors $C_{\alpha(2 n)}, n \geq 0$, are uniquely related to all on-mass-shell nontrivial spacetime derivatives of $C$ (for example, $C_{\alpha(4)}=-4 h_{\alpha \alpha}^{\mu} h_{\alpha \alpha}^{\nu} D_{0 \mu}^{L} D_{0 \nu}^{L} C$ ). Since $\mathcal{T}_{\alpha \beta}(C)$ is bilinear in $C$ we can look for $U_{\alpha \beta}(C)$ in the form

$$
\begin{aligned}
U^{\alpha \alpha}(C)= & \sigma h^{\alpha \alpha} C^{2}+\tau h_{\gamma}^{\alpha} C^{\gamma \alpha} C+\sum_{n=0}^{\infty}\left[\alpha_{n} h_{\gamma \gamma} C^{\gamma \gamma \alpha \alpha \lambda(2 n)} C_{\lambda(2 n)}\right. \\
& +\beta_{n} h_{\gamma \gamma} C^{\gamma \gamma \alpha \lambda(2 n-1)} C_{\lambda(2 n-1)}^{\alpha}+\gamma_{n} h_{\gamma \gamma} C^{\gamma \gamma \lambda(2 n)} C^{\alpha \alpha}{ }_{\lambda(2 n)} \\
& \left.+\delta_{n} h_{\gamma \gamma} C^{\gamma \alpha \alpha \lambda(2 n-1)} C_{\lambda(2 n-1)}^{\gamma}+\lambda_{n} h_{\gamma \gamma} C^{\gamma \alpha \lambda(2 n)} C_{\lambda(2 n)}^{\gamma \alpha}\right],
\end{aligned}
$$

where $\beta_{0}=\delta_{0}=0$. To prove that this is the most general Lorentz covariant form of a space-time 1-form bilinear in $C_{\alpha(2 n)}$ one has to use a simple property of the two-component spinors that the total antisymmetrization with respect to any three spinor indices gives zero. This is expressed by the identity

$$
a_{\alpha}\left(b_{\beta} c^{\beta}\right)+b_{\alpha}\left(c_{\beta} a^{\beta}\right)+c_{\alpha}\left(a_{\beta} b^{\beta}\right)=0,
$$

which is true for any three two-component spinors $a_{\alpha}, b_{\alpha}$ and $c_{\alpha}$. This identity allows one to express the terms of the type $h^{\alpha \alpha} C^{\lambda(2 n)} C_{\lambda(2 n)}, h^{\alpha}{ }_{\gamma} C^{\gamma \lambda(2 n-1)} C^{\alpha}{ }_{\lambda(2 n-1)}$, etc. via some combinations of the terms contained in (C.12).

Note that a solution of (C.11) is not unique due to the ambiguity in exact shifts $U_{\alpha \beta} \rightarrow U_{\alpha \beta}+D_{0}\left(\psi V_{\alpha \beta}\right)$, where $V_{\alpha \beta}=V_{\beta \alpha}$ is an arbitrary 0-form bilinear in $C_{\alpha(2 n)}$. The most general form of $V_{\alpha \beta}$ is

$$
V_{\alpha \beta}=\sum_{n=0}^{\infty} \rho_{n} C^{\alpha \beta \lambda(2 n)} C_{\lambda(2 n)}
$$

with arbitrary coefficients $\rho_{n}$. An interesting fact is that there exists an additional oneparametric ambiguity of the solutions of this system (we hope to come back to this issue in a future publication).

Substituting (C.12) into (C.11) and making use of (C.6) and (C.9), we arrive at some system of algebraic equations for the coefficients $\alpha_{n}, \beta_{n}, \gamma_{n}, \delta_{n}, \lambda_{n}, \sigma, \tau$. One can fix the ambiguities in exact shifts by setting

$$
\lambda_{n}=0, \quad n \geq 0, \quad \tau=0,
$$

and the afore mentioned one parametric ambiguity by setting $\alpha_{0}=0$. Then, the comparison with the explicit form of $\mathcal{T}_{\alpha \beta}(C)$ leads to the following set of relations

$$
\begin{gathered}
\alpha_{n+1}=\frac{1}{(2 n+7)\left(2 n^{2}+9 n+9\right) \lambda^{2}}\left[\frac{(2 n+5)}{2} \alpha_{n}-\frac{\left(4 n^{2}+14 n+11\right)}{(n+1)(2 n+5)} \gamma_{n}\right], \quad n \geq 1 \\
\beta_{n+1}=\frac{1}{2 i \lambda\left(2 n^{2}+9 n+9\right)}\left[(4 n+7) \gamma_{n}-\alpha_{n}\right], \quad n \geq 1, \\
\gamma_{n+1}=\frac{1}{2(2 n+5)(n+1) \lambda^{2}} \gamma_{n}, \quad n \geq 1, \\
\delta_{n}=-i \lambda\left[(2 n+5) \alpha_{n}+(2 n+1) \gamma_{n}\right], \quad n \geq 1,
\end{gathered}
$$




$$
\alpha_{1}=-\frac{47}{3360 \lambda^{3}}, \quad \beta_{1}=\frac{1}{12 i \lambda^{2}}, \quad \gamma_{1}=\frac{3}{160 \lambda^{3}}, \quad \delta_{1}=\frac{i}{24 \lambda^{2}}, \quad \sigma=-\frac{3}{8} \lambda, \quad \gamma_{0}=\frac{3}{8} \lambda,
$$

which admits a unique solution. It is therefore shown that $\mathcal{T}_{\alpha \beta}(C)$ is $D_{0}$-exact in the class of power series in higher derivatives. From the above equations it follows that the coefficients in front of higher derivatives acquire negative powers in $\lambda$, in agreement with the qualitative analysis in the beginning of this appendix. In fact, the constructed solution gives a concrete realization of the formula (C.5) in terms of the combinations of higher derivatives $C_{\alpha(n)}$. The similar phenomenon is expected to happen for higher spins. The formulae obtained in this appendix have a structure expected from the application of the integrating flow described in sect. 8 in the second order in fields.

\section{References}

[1] C. Fronsdal, Phys. Rev. D18 (1978) 3624; D20 (1979) 848.

[2] J. Fang and C. Fronsdal, Phys. Rev. D18 (1978) 3630; D22 (1980) 1361.

[3] P. van Nieuwenhuizen, Phys. Rep. 68 (1981) 189.

[4] C. Aragone and S. Deser, Phys. Lett. B86 (1979) 161.

[5] F. A. Berends, J. W. van Holten, P. van Niewenhuizen, and B. de Wit, J. Phys. A13 (1980) 1643.

[6] B. de Wit and D. Z. Freedman, Phys. Rev. D21 (1980) 358.

[7] A. K. Bengtsson, I. Bengtsson, and L. Brink, Nucl. Phys. B227 (1983) 31, 41.

[8] F. A. Berends, G. J. Burgers, and H. van Dam, Z. Phys. C24 (1984) 247; Nucl. Phys. B260 (1985) 295; B271 (1986) 429.

[9] A. K. H. Bengtsson and I. Bengtsson, Class. Quant. Grav. 3 (1986) 927.

[10] A. K. H. Bengtsson, Class. Quant. Grav. 5 (1988) 437.

[11] R. Metsaev, Mod. Phys. Lett. A6 (1991) 359.

[12] S. Coleman and J. Mandula, Phys. Rev. 159 (1967) 1251.

[13] R. Haag, J. Lopuszanski, and M. Sohnius, Nucl. Phys. B88 (1975) 257.

[14] E. S. Fradkin and M. A. Vasiliev, Phys. Lett. B189 (1987) 89; Nucl. Phys. B291 (1987) 141.

[15] M. A. Vasiliev, Phys. Lett. B285 (1992) 225 (and references therein).

[16] M. A. Vasiliev, Int. J. Mod. Phys. D5 (1996) 763.

[17] J. Maldacena, "The Large N Limit of Superconformal Field Theories and Supergravity", hep-th/9711200.

[18] S. Ferrara and C. Fronsdal, "Conformal Maxwell Theory as a Singleton Field Theory on $A D S_{5}$, IIB Branes and Duality", hep-th/9712239. 
[19] M. Gunaydin and D. Minic, "Singletons, Doubletons and $M$-theory", hepth/9802047.

[20] S. S. Gubser, I. R. Klebanov, and A. M. Polyakov, "Gauge Theory Correlators from Non-Critical String Theory", hep-th/9802109.

[21] E. Witten, "Anti De Sitter Space and Holography", hep-th/9802150.

[22] P. A. M. Dirac, J. Math. Phys. 4 (1963) 901.

[23] M. Flato and C. Fronsdal, Lett. Mat. Phys. 2 (1978) 421; Phys. Lett. B97 (1980) 236.

[24] C. Vafa, "Puzzles at Large $N "$ ", hep-th/9804172.

[25] E. Sezgin and P. Sundell, "Higher Spin $N=8$ Supergravity", hep-th/9805125.

[26] E. S. Fradkin and M. A. Vasiliev, Dokl. Acad. Nauk. 29 (1986) 1100; Ann. of Phys. 177 (1987) 63.

[27] M. A. Vasiliev, Fortschr. Phys. 36 (1988) 33.

[28] S. E. Konstein and M. A. Vasiliev, Nucl. Phys. B331 (1990) 475.

[29] M. A. Vasiliev, JeTP Lett. 50 (1989) No.8, 374; Int. J. Mod. Phys. A6 (1991) 1115.

[30] J. Moyal, Proc. Camb. Phil. Soc. 45 (1949) 99.

[31] F. Bayen, M. Flato, C. Fronsdal, A. Lichnerowicz and D. Sternheimer, Ann. Phys. (N.Y.) 110 (1978) 61, 111.

[32] A. Connes, M. R. Douglas and A. Schwarz, "Noncommutative Geometry and Matrix Theory: Compactification on Tori", hep-th/9711162.

[33] M. Douglas and C. Hull, "D-Branes and Noncommutative Torus", hep-th/9711165.

[34] M. Berkooz, "Nonlocal Field Theories and the Noncommutative Torus", hepth/9802069.

[35] N. Nekrasov and A. Schwarz, "Instantons on noncommutative $\mathbf{R}^{\mathbf{4}}$ and $(2,0)$ superconformal six dimensional theory", hep-th/9802068.

[36] C. Castro, "W Geometry From Fedosov's Deformation Quantization", hep-th/9802023.

[37] C. Devchand and O. Lechtenfeld, "Extended Self-Dual Yang-Mills from the $N=2$ String", Nucl. Phys. B516 (1998) 255, hep-th/9712043.

[38] A. Achucarro and P. K. Townsend, Phys. Lett. B180 (1986) 89.

[39] E. Witten, Nucl. Phys. B311 (1989) 46.

[40] M. P. Blencowe, Class. Quantum Grav. 6 (1989) 443.

[41] M. A. Vasiliev, Mod. Phys. Lett. A7 (1992) 3689. 
[42] H. Nicolai, Nucl. Phys. B176 (1980) 419.

[43] M. A. Vasiliev, Class. Quant. Grav. 11 (1994) 649.

[44] A. V. Barabanschikov, S. F. Prokushkin, and M. A. Vasiliev, Theor. Math. Phys. 110 (1997) 295, hep-th/9609034.

[45] R. Utiyama, Phys. Rev. D101 (1956) 1597.

[46] T. W. B. Kibble, J. Math. Phys. 2 (1961) 212.

[47] A. H. Chamseddine and P. C. West, Nucl. Phys. B129 (1977) 39.

[48] S. W. MacDowell and F. Mansouri, Phys. Rev. Lett. 38 (1977) 739.

[49] K. Stelle and P. West, Phys. Rev. D21 (1980) 1466.

[50] S. W. Hawking and D. Page, "Thermodynamics of Black Holes In Anti-de Sitter Space", Commun. Math. Phys. 87 (1983) 577.

[51] E. P. Wigner, Phys. Rev. 77 (1950) 711.

[52] F. A. Berezin and M. A. Shubin, "Schrödinger Equation", Moscow Univ. Press, Moscow, 1983.

[53] M. A. Vasiliev, Class. Quantum Grav. 8 (1991) 1387.

[54] M. A. Vasiliev, Proceedings of the Second International Workshop on Selected Topics of Theoretical and Modern Mathematical Physics, Tbilisi, Georgia, September 22-28, Eds: M. Eliashvili, G. Jorjadze, E. Ragoucy and P. Sorba, ENSLAPP-A-642/97.

[55] M. A. Vasiliev, Nucl. Phys. B (Proc. Suppl.) 56B (1997) 241.

[56] M. A. Vasiliev, "Deformed Oscillator Algebras and Higher Spin Gauge Interactions of Matter Fields in 2+1 Dimensions", hep-th/9712246.

[57] D. A. Kirzhnitz, JETP 49 (1965) 1544; in Sbornik "Problems of Theoretical Physics" in memory of I. E. Tamm, Moscow, "Nauka", 1972.

[58] M. A. Vasiliev, Ann. Phys. (N.Y.) 190 (1989) 59.

[59] M. Green, J. Schwarz, E. Witten, "Superstring Theory", vols. 1 and 2, Cambridge Univ. Press, New York, 1987.

[60] E. S. Fradkin and M. A. Vasiliev, Int. J. Mod. Phys. A3 (1988) 2983.

[61] E. Bergshoeff, B. de Wit, and M. A. Vasiliev, Nucl. Phys. B366 (1991) 315.

[62] B. de Wit, A. K. Tollstén, and H. Nicolai, Nucl. Phys. B392 (1993) 3, hepth/9208074.

[63] H. Nicolai, E. Sezgin, and Y. Tanii, Nucl. Phys. B305 [FS23] (1988) 483.

[64] E. Janke, F. Emde, F. Lösh, Tafeln Höherer Funktionen, B. G. Teubner Verlagsgesellsehaft, Stuttgart, 1960. 